\title{
Colloquium: Atomic spin chains on surfaces
}

\author{
Deung-Jang Choi $\odot$ \\ Centro de Física de Materiales CFM/MPC (CSIC-UPV/EHU), \\ Paseo Manuel de Lardizabal 5, 20018 Donostia-San Sebastián, Spain, \\ Donostia International Physics Center (DIPC), \\ Paseo Manuel de Lardizabal 4, 20018 Donostia-San Sebastián, Spain, \\ and Ikerbasque, Basque Foundation for Science, 48013 Bilbao, Spain
}

\author{
Nicolas Lorente \\ Centro de Física de Materiales CFM/MPC (CSIC-UPV/EHU), \\ Paseo Manuel de Lardizabal 5, 20018 Donostia-San Sebastián, Spain \\ and Donostia International Physics Center (DIPC), \\ Paseo Manuel de Lardizabal 4, 20018 Donostia-San Sebastián, Spain
}

Jens Wiebe and Kirsten von Bergmann

Department of Physics, University of Hamburg, D-20355 Hamburg, Germany

\author{
Alexander F. Otte \\ Department of Quantum Nanoscience, Kavli Institute of Nanoscience, \\ Delft University of Technology, Lorentzweg 1, 2628 CJ Delft, The Netherlands
}

\author{
Andreas J. Heinrich (1) \\ Center for Quantum Nanoscience, Institute for Basic Science (IBS), \\ Seoul 03760, Republic of Korea \\ and Department of Physics, Ewha Womans University, \\ Seoul 03760, Republic of Korea
}

\section{(published 4 October 2019)}

\begin{abstract}
Magnetism at low dimensions is a thriving field of research with exciting opportunities in technology. This Colloquium focuses on the properties of 1D magnetic systems on solid surfaces. From the emulation of 1D quantum phases to the potential realization of Majorana edge states, spin chains are unique systems to study. The advent of scanning tunneling microscope (STM) based techniques has permitted us to engineer spin chains in an atom-by-atom fashion via atom manipulation and to access their spin states on the ultimate atomic scale. Here the current state of research on spin correlations and dynamics of atomic spin chains as studied by the STM is presented. After a brief review of the main properties of spin chains on solid surfaces, spin chains are classified according to the coupling of their magnetic moments with the holding substrate. This classification scheme takes into account that the nature and lifetimes of the spin-chain excitations intrinsically depend on the holding substrate. Interest is shown of using insulating layers on metals, which generally results in an increase in the spin state's lifetimes such that their quantized nature gets evident and they are individually accessible. Next shown is the use of semiconductor substrates promising additional control through the tunable electron density via doping. When the coupling to the substrate is increased for spin chains on metals, the substrate conduction electron mediated interactions can lead to emergent exotic phases of the coupled spin chain-substrate conduction electron system. A particularly interesting example is furnished by superconductors. Magnetic impurities induce states in the superconducting gap. Because of the extended nature of the spin chain, the in-gap states develop into bands that can lead to the emergence of $1 \mathrm{D}$ topological superconductivity and consequently to the appearance of Majorana edge states. Finally, an outlook is given on the use of spin chains in spintronics, quantum communication, quantum computing, quantum simulations, and quantum sensors.
\end{abstract}

DOI: 10.1103/RevModPhys.91.041001

\section{CONTENTS}

I. Introduction

II. Concepts of Spin-chain Physics
A. Spin Hamiltonians

B. Excitations in spin chains

C. Haldane phase

D. Decoherence of spin chains 
III. Experimental Methods and Sample Systems

A. Experimental methods

B. Preparation of chains on surfaces

C. Spin chains and their holding substrates

IV. Spin Chains Decoupled from the Substrate's

Electron Bath

A. Passivated metal substrates

B. The impurity problem and its extension to spin chains

C. Semiconductor substrates

V. Spin Chains Strongly Coupled to the Substrate's Electron Bath

A. Spin chains on metallic substrates

B. Spin chains on superconducting substrates

VI. Outlook

Acknowledgments

References

\section{INTRODUCTION}

A collection of local magnetic moments arranged in a linear fashion that interact via some spin-spin coupling is generally known as a spin chain. This seemingly simple object is one of the most complex and rich physical systems that have been studied since the advent of quantum mechanics without a decline in interest ever since. As early as 1928, Werner Heisenberg explained ferromagnetism using Pauli's exclusion principle and the interaction between spins that bears his name. Subsequently, antiferromagnetism was addressed in spin chains by the seminal works of Bethe (1931) and Hulthén (1938). Also in recent times the interest in spin chains continues. The 2016 Nobel Prize explicitly mentioned spin chains through the work of Haldane $(1983,2017)$ that revolutionized the understanding of condensed-matter physics by finding new phases of matter associated with a certain set of the two interactions defining the spin-chain parameters (Haldane, 1983, 2017; Affleck, 1989). Additionally, the study of spin chains has been instrumental in ushering the farreaching concepts of topology in condensed matter.

Spin chains are also paradigmatic integrable systems. Bethe developed the Bethe ansatz to solve antiferromagnetically coupled spin chains (Bethe, 1931; Hulthén, 1938), which has found use in many other integrable models (Sklyanin and Faddeev, 1978; Takhtajan, 1981; Faddeev, 1996).

The simplification of spin chains as compared to threedimensional systems brings in new phenomena proper to lower dimensions. One of them is the absence of long-range order as descibed by the Mermin-Wagner theorem (Mermin and Wagner, 1966). A related consequence is that phase transitions in one-dimensional (1D) systems take place only at zero kelvin. Furthermore, correlations are enhanced at 1D. As a consequence, many-body physics is ubiquitous in 1D systems.

While the initial interest in spin chains was primarily from a theoretical viewpoint, various ways exist to create physical realizations of spin chains in either solids, trapped atoms, or molecules. Particularly the development of the scanning tunneling microscope (STM) has furthered permitted us to create spin chains on solid surfaces with atomic precision.

The first experimental realizations of spin chains date from the early 1960s. It was found that some transition metal (TM) salts had their magnetic centers arranged in a chainlike fashion and showed exchange interactions between these centers (Watanabe and Haseda, 1958; Haseda and Miedema, 1961; Flippen and Friedberg, 1963; Wagner and Friedberg, 1964). An interesting family of 1D spin systems is based on $\mathrm{Cu}$ ions (Sahling et al., 2015). Recent activity is moving instead into the creation of extraordinary spin chains using molecular systems (Caneschi et al., 2001; W.-X. Zhang et al., 2013).

A great deal of progress in the experimental investigation of the physics of spin chains has been achieved in developing quantum simulators based on atomic traps. Spin interactions can be simulated by the close-ranged interactions between atoms held in an optical trap (Simon et al., 2011). When strongly interacting multicomponent gases are arranged in one dimension, effective Heisenberg spin chains can be modeled (Deuretzbacher et al., 2014). Short-ranged strong interactions between alkali atoms have also been used to simulate the Heisenberg $X X Z$ models (Volosniev et al., 2015; Yang and Cui, 2016). More recently, simulations of antiferromagnetic Heisenberg spin chains have been performed using four fermionic atoms (Murmann et al., 2015). Also Yang, Guan, and Cui (2016) showed that these floating atoms can lead to other interesting examples of Heisenberg chains.

The present Colloquium is devoted to the study of spin chains on solid surfaces as studied with the STM. The STM allows us to interrogate matter on the atomic scale with unprecedented precision. Besides studying spin chains built by self-assembling techniques, the STM can actively displace, transfer, and position atoms on a solid surface (Eigler and Schweizer, 1990). In this way, spin chains can be built with atomic precision of both the chain itself as well as its environment. Furthermore, recent progress has permitted us to greatly enhance the applications of STM by conferring it with the ability of measuring single-atom magnetic excitations (Heinrich et al., 2004), single-atom magnetization curves (Meier et al., 2008), single-atom fast time-resolved spin dynamics (Loth, von Bergmann et al., 2010), and singleatom electron paramagnetic resonances (Baumann, Paul et al., 2015). With these new capabilities, the spin chains can be assembled and characterized atom by atom with a unique combination of control and accuracy. As a consequence a new world of data is booming in the field of spin chains.

The recent years have seen a great deal of activity in the field of spin chains on solids. We review this activity classifying the STM-based research by the substrate system. This allows us to review processes as interesting as Kondo physics in heterogeneous spin chains (Choi, Robles et al., 2017) or as ground breaking as the observation of indications for Majorana edge states in condensed matter (Nadj-Perge et al., 2014; Kim et al., 2018).

\section{CONCEPTS OF SPIN-CHAIN PHYSICS}

The extraordinary interest in spin chains stems from their complex quantum nature. In this section, we review the properties of spin chains by first deriving simplified Hamiltonians that consider only effective interactions among magnetic moments. Next we study the excitation spectra of these effective Hamiltonians, first by considering the Heisenberg model and then the effect of magnetic anisotropy. Finally, we analyze the complexity of these solutions by 
revealing the role of entanglement, comparing it with manybody correlations and explaining the effect of decoherence of spin chains on solid surfaces.

\section{A. Spin Hamiltonians}

The Hamiltonian of a nonrelativistic atomic system representing, for instance, a condensed-matter realization of a chain of spins does not contain any spin operator because the spin is contained in the electronic states. As a consequence, the total spin operator $\left(\hat{S}^{2}\right)$ and one of the components of the spin (say $\hat{S}_{z}$ ) will commute with the Hamiltonian. When relativistic effects are included, the spin operator fully appears in the spinorbit coupling terms, and both $S$ and $S_{z}$ can cease to be good quantum numbers.

Heisenberg Hamiltonian.-Spin operators naturally appear in a Hamiltonian if we simplify matters to include only the low-energy excitations of the full system. Generally, magnetic excitations are of low energy and a spin Hamiltonian will explicitly consider them.

Open-shell atoms have two sources of magnetic moment $\hat{\vec{L}}$ and $\hat{\vec{S}}$ that add to give the magnetic moment $\hat{\vec{M}}=$ $-\mu_{B}(\hat{\vec{L}}+2 \hat{\vec{S}})$. Here we restrict ourselves to spins in a vague way, but they can be any of the operators that contribute to the magnetic moment of the system. The aim of the spin Hamiltonian is to simplify the description of the magnetic structure of the system by using effective interactions among spins. The Heisenberg Hamiltonian is a clear case of a spin Hamiltonian. It is a simple model for the interaction between two magnetic moments. The actual interaction behind electrons giving rise to the effective interaction can be quite intricate. Take, for example, the superexchange interaction between two localized orbitals 1 and 2 [see Yosida (2001) for more details]. The original Hamiltonian includes only a nearest-neighbor hopping term $t$, that leads to chemical hybridization, and an on-site Coulomb repulsion term $U$ that adds a penalty to double occupations of some local orbitals. The low-energy excitations can be represented by the solutions of a Heisenberg Hamiltonian with an antiferromagnetic interaction given by

$$
\hat{H}_{\text {Heisenberg }}=J \hat{\vec{S}}_{1} \cdot \hat{\vec{S}}_{2} .
$$

The coupling term is given by (Yosida, 2001)

$$
J=\frac{2 t^{2}}{U} .
$$

In order to take into account the varying nature of different localized magnetic moments, we can generalize the Heisenberg Hamiltonian to

$$
\hat{H}=\frac{1}{2} \sum_{i j} \hat{\vec{S}}_{i} \cdot \mathrm{J}_{i j} \cdot \hat{\vec{S}}_{j}
$$

with a full magnetic exchange tensor $\mathrm{J}_{i j}$ that takes into account all couplings between different pairs of effective spins $\hat{\vec{S}}_{i}, \hat{\vec{S}}_{j}$ of localized magnetic moments $i$ and $j$.
This operator can be separated into three contributions (Udvardi et al., 2003; Hermenau et al., 2019):

$$
\begin{aligned}
\hat{H}= & \frac{1}{2} \underbrace{\sum_{i \neq j} J_{i j} \hat{\vec{S}}_{i} \cdot \hat{\vec{S}}_{j}}_{\text {isotropic exchange }}+\underbrace{\frac{1}{2} \sum_{i \neq j} \vec{D}_{i j} \cdot\left(\hat{\vec{S}}_{i} \times \hat{\vec{S}}_{j}\right)}_{\text {Dzyaloshinskii-Moriya interaction }} \\
& +\underbrace{\frac{1}{2} \sum_{i \neq j} \hat{\vec{S}}_{i} \cdot J_{i j}^{\text {aniso }} \cdot \hat{\overrightarrow{\vec{S}}}_{j} .}_{\text {anisotropic exchange }}
\end{aligned}
$$

The tensor of exchange interactions $\mathbf{J}_{i j}$ was split into its constituents: the isotropic exchange interaction $J_{i j}$, the DzyaloshinskiiMoriya interaction (DMI) $\vec{D}_{i j}$, and the symmetric anisotropic exchange interaction $\mathrm{J}_{i j}^{\mathrm{aniso}}=\left[\mathrm{J}_{i j}+\left(\mathrm{J}_{i j}\right)^{\mathrm{T}}\right] / 2-J_{i j}$.

The DMI can arise when the inversion symmetry of a system with sizable spin-orbit coupling is broken, becoming one source of noncollinear arrangements of spins (Dzyaloshinsky, 1958; Moriya, 1960; Fert and Levy, 1980; Levy and Fert, 1981). The DM vector $\vec{D}_{i j}$ gives the strength and orientation of the interaction and is subject to symmetry selection rules; this interaction minimizes the energy for an orthogonal orientation of adjacent spins and dictates the rotational sense of the spin vectors. In competition with the isotropic and anisotropic Heisenberg exchange, it can lead to ground states that are spin spirals exhibiting a unique rotational sense (Menzel et al., 2012; Schweflinghaus et al., 2016). The DMI is also an important ingredient for the formation of magnetic skyrmions in two dimensions (Heinze et al., 2011).

All the different parts of the exchange interactions in Eq. (4) can in principle not only result from the super exchange previously discussed, but also from the direct exchange for close distance between the two localized spin-carrying orbitals, or from the conduction electron mediated indirect exchange interaction for a further separation of the localized orbitals. The isotropic part of the latter type of interaction is known as the Ruderman-Kittel-Kasuya-Yosida (RKKY) interaction (Ruderman and Kittel, 1954; Kasuya, 1956; Yosida, 1957). It typically has a damped oscillatory dependence, i.e., it changes between ferromagnetic and antiferromagnetic coupling as a function of the distance between two atomic spins and their orientation with respect to the substrate lattice. The latter behavior results from the shape of the Fermi surface of the conductance electrons that can be rather complex and anisotropic (Zhou et al., 2010). Because of the inversion symmetry breaking due to the presence of a surface, the conduction electron mediated exchange interaction also has a Dzyaloshinskii-Moriya contribution that can be as large as the isotropic contribution if the substrate electrons are subject to considerable spin-orbit interaction (Smith, 1976; Fert and Levy, 1980; Khajetoorians et al., 2016). As a result, chains of indirect conduction electron exchange coupled atoms on high atomic number metallic substrates can also have spin-spiral ground states (Steinbrecher et al., 2018).

Finally, there can be higher-order terms of the exchange interaction. The next higher order involves hopping between 
four-spins, located on two, three, or four sites (Kurz et al., 2001; Blügel and Bihlmayer, 2007; Hoffmann and Blügel, 2018).

Magnetic anisotropy.-So far, only the interactions between localized magnetic moments have been considered in Eq. (4). However, the electronic orbitals also interact with the surrounding ligands via Coulomb interactions. Together with spin-orbit coupling, this leads to a dependence of the system's energy on the orientation of the magnetic moment, the so-called on-site magnetic anisotropy. In order to take this into account, an additional contribution is added to the Hamiltonian where the orbital degrees of freedom of the electronic wave function are implicit and only the spin degrees of freedom are actively considered. For the sake of understanding we first consider a low-symmetry binding site which leads to sufficient splitting of the orbital degrees of freedom.

In the absence of spin-orbit interaction, if the value of the orbital angular momentum contribution to the magnetic moment is negligible, a particularly simple case takes place. This is often the case when the symmetry is strongly broken by the substrate holding the magnetic atoms. Because of this quenching of the orbital magnetic moment, the low-energy excitations of the effective spin Hamiltonian are free of active orbital transitions when the spin-orbit interaction is connected. However, despite the quenching of the orbital moment, there will be a final nonzero value of the orbital moment due to the efficient mixing of spin and orbital degrees of freedom by the spin-orbit interaction.

The lowest-order terms in the additional magnetic anisotropy contribution to the effective spin Hamiltonian correspond to uniaxial symmetry of the ligand field and, allowing for some nontrivial symmetry transversal to the main axis (such as $C_{i}$, $C_{s}, C_{2 \nu}$, etc.), have the form

$$
\hat{H}=D \hat{S}_{z}^{2}+E\left(\hat{S}_{x}^{2}-\hat{S}_{y}^{2}\right)
$$

This Hamiltonian is found very often, as, for example, in the case of magnetic impurities on $\mathrm{Cu}_{2} \mathrm{~N}$ surfaces as will be described in Sec. IV.A. Other ligand or crystal symmetries lead to the survival or canceling of higher powers of the spin operators. The Stevens operators are a systematic way to include contributions to the spin Hamiltonian taking into account the symmetry of the atomic environment (Stevens, 1952; Rudowicz and Chung, 2004). Stevens generalized the spin Hamiltonian to read

$$
\hat{H}=\sum_{k=2,4,6} \sum_{q=-k}^{k} B_{k}^{q} \hat{O}_{k}^{q}(\vec{S})
$$

Each of the $\hat{O}_{k}^{q}(\vec{S})$ operators is Hermitian and the coefficients $B_{k}^{q}$ are real such that the spin Hamiltonian is Hermitian. A rank $k$ of 6 is sufficient to describe the effects of crystal-field symmetry on spins on surfaces.

The axial anisotropy can be expressed using the Stevens coefficients $B_{k}^{q}$ for the zero-field splitting parameters, such that

$$
D=3 B_{2}^{0}, \quad E=B_{2}^{2},
$$

where $k=2$ implies that they refer to axial symmetry and $q=0,2$ refer to the longitudinal and transversal components, respectively. The corresponding Stevens operators are

$$
\hat{O}_{2}^{0}=3 \hat{S}_{z}^{2}-S(S+1)
$$

where $S$ is the spin eigenvalue and $\hat{S}_{z}$ is the $z$ component of the spin operator. And

$$
\hat{O}_{2}^{2}=\hat{S}_{x}^{2}-\hat{S}_{y}^{2}
$$

The Stevens operators are widely tabulated and can be found in many references, for example, Rudowicz and Chung (2004) and references therein.

Many substrates possess a $C_{3 \nu}$ symmetry. An example is the substrates of Sec. IV.A. In many instances, we see that higherorder terms can often be neglected, such that using $D \hat{S}_{z}^{2}$ is already good enough for those systems.

Writing such an effective spin Hamiltonian is not always possible. In the absence of quenching of the orbital degrees of freedom, the spatial dependence of the electronic wave function has to be explicitly allowed in the Hamiltonian. This case has been considered in a number of recent works about magnetic impurities on a $\mathrm{MgO}$ thin film on a $\mathrm{Ag}(100)$ substrate (Rau et al., 2014; Baumann et al., 2015; Baumann, Paul et al., 2015; Ferrón, Delgado, and Fernández-Rossier, 2015). These articles study $3 d$ transition metals on $\mathrm{MgO}$ layers. Generally, adsorption on one of the surface's oxygen atoms is preferred, leading to an axial symmetry given by the normal to the surface, plus a fourfold symmetry by the four neighboring $\mathrm{Mg}^{2+}$ ions. The axial crystal field is not strong enough to sufficiently quench the orbital moment and a full multiplet calculation must be undertaken. Figure 1 shows the typical procedure to obtain the low-energy terms of a $\mathrm{Co}^{2+}$ ion on $\mathrm{MgO}$. The calculations proceed by first considering the axial field effect on the electronic states of the studied $3 d$ ion.

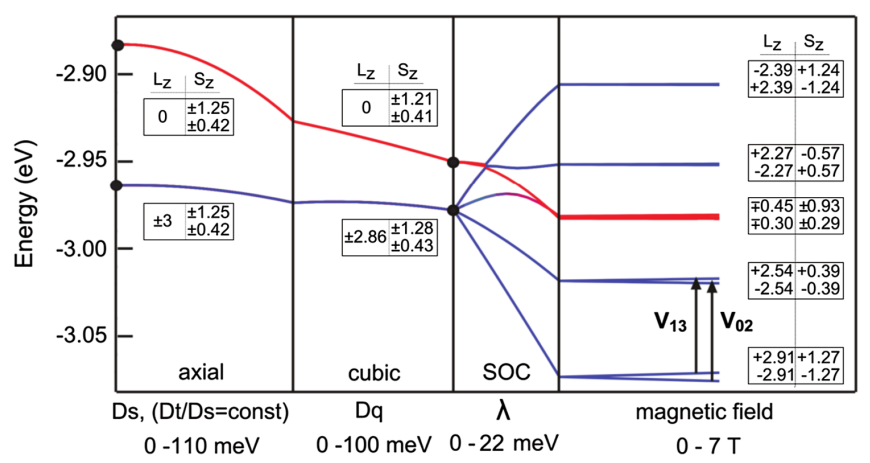

FIG. 1. The effect of different perturbations on the electronic states of $\mathrm{a} \mathrm{Co}^{2+}$ ion on $\mathrm{MgO}$ is shown incrementally. First the axial field due to the presence of the surface plane is included, shifting the ten low-energy spherical levels (one eightfold and one twofold degenerate), and second the crystal field of the four neighboring $\mathrm{Mg}^{2+}$ ions is considered. Next the spin-orbit coupling is adiabatically switched on. And finally a magnetic field is included. The lowest-energy transitions induced by tunneling electrons from an STM are depicted by arrows. From Rau et al., 2014. 
Next the fourfold crystal field is added. Once the electronic states reflect the correct orbital structure under the environment's fields, the spin-orbit interaction is added. Finally the Zeeman splitting due to an external magnetic field is considered. This approach incrementally considers each effect permitting us to gain insight as well as control on the actual electronic configuration of the ion in its environment. By this procedure, a spin Hamiltonian enhanced by orbital terms, similar to the spin terms, can be obtained that reproduces the low-energy states of the system.

Classical magnetic moments. - If the quantum properties of the spin-chain system are not dominating, e.g., because of very large spin values, it is often sufficient to consider the classical limit. Within this limit, the vector spin operator $\hat{\vec{S}}$ is replaced by a classical magnetic moment, via $\vec{m}=-g \mu_{B} \vec{S}$, and the parameters within the Stevens operator treatment are related to classical magnetic anisotropy constants.

\section{B. Excitations in spin chains}

The ground state of a ferromagnetic Heisenberg chain in the presence of an arbitrarily small magnetic field corresponds to all spins being aligned along the magnetic field. Flipping a spin does not result in an eigenstate of the Hamiltonian; instead, it forms a linear combination of eigenstates (Auerbach, 1994; Yosida, 2001; Mattis, 2006). Since the complete reversal of a single spin requires a lot of energy due to the exchange interaction, the lowest-energy excitations of chains are spin waves, where the spin flip is delocalized with a phase shift along the entire chain. For an infinite chain of $S=1 / 2$ spins, a spin-wave excitation has the following dispersion relation:

$$
\epsilon(\vec{q})=2 J \sin ^{2}\left(\frac{\vec{a} \cdot \vec{q}}{2}\right),
$$

where $\vec{q}$ is the dispersion momentum vector along the infinite spin chain and $\vec{a}$ is the lattice vector of the spin chain. Spin waves are also referred to as magnons.

If the Heisenberg coupling between spins is instead antiferromagnetic, the spin states are far from simple due to the multiconfigurational character of the antiferromagnetic solutions. For chains of atoms with $S=1 / 2$, the flipping of one spin leads to either a spin wave or a two-spinon excitation. Either of these excitations changes the total magnetization by $\Delta S= \pm 1$. The spin wave is the lowest-energy excitation of the antiferromagnetic chain and its dispersion relation is given by (des Cloizeaux and Pearson, 1962)

$$
\epsilon(\vec{q})=\frac{\pi}{2} J|\sin (\vec{a} \cdot \vec{q})|,
$$

with the same notation as before.

Calculations on the probability and spectra of finite ferromagnetic and antiferromagnetic spin chains show that spin waves are efficiently excited by tunneling electrons (Gauyacq and Lorente, 2011).

Just beyond the spin-wave excitation two-spinon excitations set in, until they reach the upper boundary (Yamada, 1969; Müller et al., 1981; Karbach et al., 1997; Bougourzi, Karbach, and Müller, 1998; Karbach, Hu, and Müller, 1998)

$$
\epsilon_{U}(\vec{q})=\pi J|\sin (\vec{a} \cdot \vec{q} / 2)|
$$

Figure 2(a) shows the continuum of two-spinon excitations bounded by the spin-wave excitation, Eq. (8), and the upper branch, Eq. (9). All these excitations correspond to encountering one spin flip in an antiferromagnetic spin chain. Half an excitation is a spinon, which is a consequence of the tendency to the fragmentation of spin (and charge) in 1D systems (Mourigal et al., 2013). This type of spectrum was recently revealed in 1D spin chains formed by $\mathrm{CuO}$ (Mourigal et al., 2013). The fragmentation of spin in the excited state is easily understood when the time evolution of the two spinons is followed. Figure 2(b) shows a simple scheme of the creation of a two-spinon excitation and its time evolution into single spinons. The two-spinon continuum is followed by fourspinon excitations and so forth at even higher energies (Müller et al., 1981; Caux and Hagemans, 2006), but most of the spectral weight is carried by the two-spinon excitations (Müller et al., 1981; Karbach et al., 1997).

\section{Haldane phase}

For a while, it was believed that the excitation spectra for spin $1 / 2$ systems was general to all antiferromagnetic Heisenberg spin chains. However, Haldane (1983) predicted that the spectra for chains made out of integer spins $(S \geq 1)$ should be gapped, meaning that there are no zero-energy excitations contrary to the spectra shown in Fig. 2(a).

Haldane conjectured that the Heisenberg antiferromagnetic spin chain for integer spins has a singlet ground state separated by an energy gap from the first excited state (Haldane, 1983); see also Affleck (1989) and Tasaki (1991). This apparently minor difference has important implications. The appearance

(a)

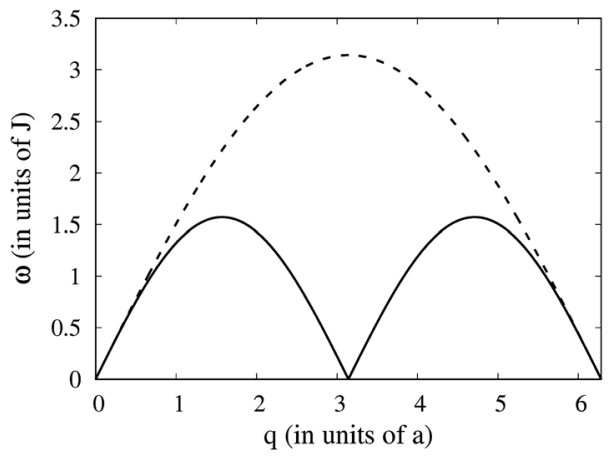

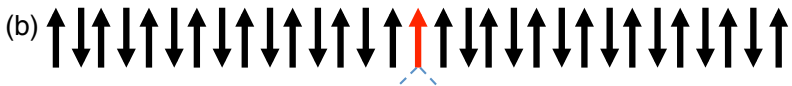

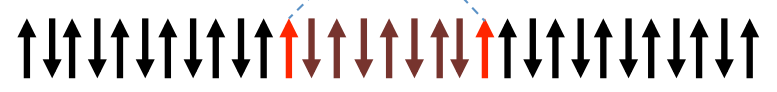

FIG. 2. (a) Two-spinon continuum corresponding to single spinflip excitations of an inifinite Heisenberg antiferromagnetic chain of atoms with $S=1 / 2$ (spin $1 / 2$ chain). The continuum is bounded by spin-wave excitations as the low-energy branch, Eq. (8), and the higher branch, Eq. (9). (b) Scheme of a spin 1/2 chain showing the propagation of a two-spinon becoming two domain-wall excitations for an Ising antiferromagnetic chain. 
of the gap leads to spin-spin correlations that decay exponentially with distance while for the half-integer the spin-spin correlations decay following a power law (Tasaki, 1991; Renard, Regnault, and Verdaguer, 2002). But moreover, it leads to the possibility of nontrivial ground states for the integer case with a corresponding topological quantum phase transition between the different solutions ( $\mathrm{Gu}$ and Wen, 2009; Pollmann et al., 2010; Turner, Pollmann, and Berg, 2011; Pollmann, 2018). For a chain of $S=1$ spins, the Heisenberg antiferromagnetic spin chain with uniaxial anisotropy $[E=0$ in Eq. (5)] presents a phase transition for $D=J[J$ and $D$ defined in Eqs. (1) and (5)]. Both states on either side of the phase transition preserve all the symmetries of the Hamiltonian; hence the phase transition does not take place by breaking symmetries, but it is rather of a topological nature (Chen et al., 2012). The low $D$ phase is the Haldane phase that is a strongly entangled state that cannot be smoothly connected to a product state. However, the large $D$ phase can be connected to a simple product state. This last phase is the topologically trivial one (Pollmann, 2018).

The confirmations of the integer spin system being gapped are quite limited, despite all the existing experimental work on 1D spin systems (Renard et al., 1987; Renard, Regnault, and Verdaguer, 2002). Indeed, the requirements to obtain the Haldane phase are somewhat difficult to find in a physical system. The individual spins must be integers, the interaction antiferromagnetic, arranged in 1D periodical structures with uniform interactions, but weak interchain interactions and weak anisotropy. Renard, Regnault, and Verdaguer (2002) gave a complete list of Ni-based compounds with quasi-1D spin-one chains that present the Haldane phase.

An extension of Heisenberg spin chains is given by the AKLT model named after Affleck, Lieb, Kennedy, and Tasaki (Affleck et al., 1987). The AKLT model consists of a chain of sites that are connected by a bond. This valence bond contains two spins $1 / 2$. Then each site is effectively a spin 1 system, but due to the valence bond that is singlet, the sites are antiferromagnetically coupled. This model can be written into a spin Hamiltonian by using projectors, arriving at the following expression:

$$
\hat{H}=\sum_{j}\left(\hat{\vec{S}}_{j} \cdot \hat{\vec{S}}_{j+1}+\frac{1}{3}\left(\hat{\vec{S}}_{j} \cdot \hat{\vec{S}}_{j+1}\right)^{2}\right)
$$

which is a spin 1 Heisenberg Hamiltonian plus an extra biquadratic term. This model is exactly solvable, and its ground state can be expressed as a matrix product state which still stirs a lot of theoretical attention. Furthermore, the model was used to study valence-bond order and symmetryprotected topological order ( $\mathrm{Gu}$ and Wen, 2009; Pollmann et al., 2012).

\section{Decoherence of spin chains}

In the following, we discuss decoherence effects that arise by the interaction of the spin chain with the environment, which in this work is the holding substrate.

Let us first assume we have two $S=1 / 2$ spins interacting via an exchange interaction $J \vec{S}_{1} \cdot \vec{S}_{2}$. We can diagonalize this Hamiltonian and obtain three $S=1$ states and one $S=0$ state. If we measure one of the spins, we will find equal probabilities for spins up and down. Thus, we cannot obtain any information on the state of an individual spin. However, we know the total spin of the two-spin system. It is perfectly determined. The total states are canonical maximally entangled states (Horodecki et al., 2009). Once we know the state of the full system, and the state of one of the spins, we will know the outcome of a possible measurement on the other spin.

This is true while the spins keep their respective relative phases. In the events of collisions or perturbations that simply produce a change of phase on one of the components, the wave function changes and the previous entangled wave function does not represent the system anymore. Indeed, for long enough times, the accumulation of dephasing events leads to the collapse of the singlet wave function in either $|\uparrow \downarrow\rangle$ or $|\downarrow \uparrow\rangle$, also known as the Néel states. When the spin chain is in contact with a substrate, statistical interactions with the substrate (phonon or electron collisions) lead to dephasing and hence decoherence.

In the case of spin chains on surfaces, the effect of decoherence has been shown to lead to Néel-like solutions of antiferromagnetic spin chains (Gauyacq and Lorente, 2015; Delgado and Fernández-Rossier, 2017). It is instructive to compare the cases of $\mathrm{Fe}_{x}$ (Loth et al., 2012) and $\mathrm{Mn}_{x}$ (Hirjibehedin, Lutz, and Heinrich, 2006; Choi, Robles, Gauyacq, Rubio-Verdú et al., 2016) spin chains. The main difference of these two systems is the magnetic anisotropy, Eq. (5). The spin on $\mathrm{Fe}$ atoms on $\mathrm{Cu}_{2} \mathrm{~N}$ shows a large anisotropy, while Mn displays a very small one. As a consequence, the atomic spin of Fe has a preferential direction where it can easily align and create Néel-like states with aid from the environmental decoherence. Even for similar decoherence rates, the absence of a preferential axis for Mn makes it more difficult to collapse into a classical Néel state.

The time evolution of the density matrix can be obtained from the time evolution of the system state. The density matrix is an operator given by the projector on the state of the full system $|\Psi\rangle$, then the density matrix is $\hat{\rho}=|\Psi\rangle\langle\Psi|$. The time evolution leads to

$$
\frac{d \hat{\rho}}{d t}=-\frac{i}{\hbar}[\hat{H}, \hat{\rho}] .
$$

Let us assume that we can express the total-system Hilbert space as the direct product of the two subsystem Hilbert space $\mathcal{H}=\mathcal{H}_{A} \otimes \mathcal{H}_{B}$, where, for example, $A$ can stand for the spin chain and $B$ for the holding substrate. Once we have determined $H_{B}$, we can use an eigenstate basis

$$
\hat{H}_{B}\left|\phi_{B}\right\rangle=\epsilon_{B}\left|\phi_{B}\right\rangle
$$

to project out the $B$ subsystem, leading to the reduced density matrix

$$
\hat{\rho}_{A}=\sum_{B}\left\langle\phi_{B} \mid \Psi\right\rangle\left\langle\Psi \mid \phi_{B}\right\rangle .
$$

When the reduced density matrix is used, new terms explicitly appear in the time-evolution equation. This can be written in 
terms of the dissipative part of the Liouvillian $\mathcal{L}$ (CohenTannoudji, Dupont-Roc, and Grynberg, 1998). The actual way of doing this is very involved and many works treat this problem (Cohen-Tannoudji, Dupont-Roc, and Grynberg, 1998; Delgado and Fernández-Rossier, 2017)

$$
\frac{d \hat{\rho}_{A}}{d t}=-\frac{i}{\hbar}\left[\hat{H}_{A}, \hat{\rho}_{A}\right]+\mathcal{L}\left(\hat{\rho}_{A}\right)
$$

The effect of the environment amounts to a source of random interactions between the many degrees of freedom of the environment (subsystem $B$ ) and the degrees of freedom of subsystem $A$. The Liouvillian can then be approximated by a linear term on the differential equation for the coherences with a decay constant $1 / T_{2}^{*}$. Here $1 / T_{2}^{*}$ is the pure decoherence or pure dephasing rate. Let us assume that we have only two states ( 1 and 2 ), and then the dissipative part of the Liouvillian $\mathcal{L}$ is simply

$$
\mathcal{L}\left(\hat{\rho}_{A}\right)=-\frac{1}{T_{2}^{*}}\left\{\hat{\rho}_{12}+\hat{\rho}_{21}\right\}
$$

Here we assumed no direct transition between states such that $1 / T_{1}=0$. For more states, matrices have to be defined for the dephasing rates and the equations become considerably more difficult without changing the physics. A complete account of the quantum dynamics of a magnetic subsystem can be found in Delgado and Fernández-Rossier (2017).

The above equations (14) and (15) found direct application in the problem of the quantum dynamics of a spin chain. The experiment by Loth et al. (2012) consisted of assembling an antiferromagnetic Fe chain on $\mathrm{Cu}_{2} \mathrm{~N}$. The spin-polarized STM image allowed them to measure the dwelling times in each of the two Néel states of the spin chain. They found that at very

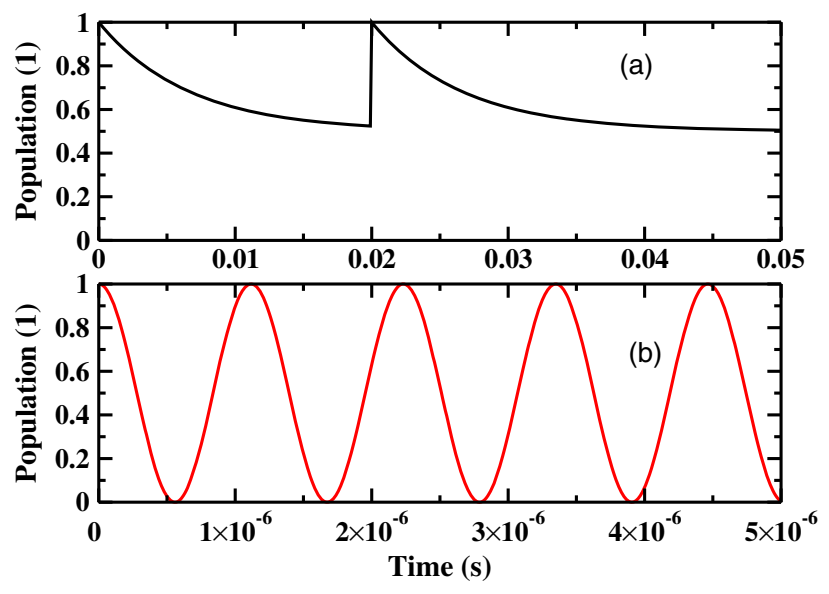

FIG. 3. $\quad \mathrm{A} \mathrm{Fe}_{6}$ chain is initially in state 1 of the two classical Néel states of this antiferromagnetic spin chain. In the presence of decoherence, the population of state $1\left(\rho_{11}\right)$ is exponentially reduced to 0.5 , populating both states, (1), while the coherences become zero $\left(\rho_{12}=\rho_{21} \rightarrow 0\right)$. Here a measurement at $\sim 0.02 \mathrm{~s}$ is assumed to find the system in state 1 , and then the population is suddenly $100 \%$ for state 1 . Afterward the exponential decay leads to $50 \%$ populations. In the absence of decoherence, the population is given by Rabi oscillations of very fast frequency for the $\mathrm{Fe}_{6}$ chain on $\mathrm{Cu}_{2} \mathrm{~N}$. From Gauyacq and Lorente, 2015. low temperatures the switching rate between the two states was a constant with temperature.

Calculations based on this formalism showed that the spinchain dynamics was a competition between quantum tunneling, which leads to Rabi oscillations between the two Néel states, and the decaying effect of decoherence (Gauyacq and Lorente, 2015). Pure quantum tunneling dynamics leads to fast oscillations of the state populations. However, due to decoherence, the population evolution becomes exponential. Figure 3 shows the difference between the spin-chain dynamics under decoherence [Fig. 3(a)], or quantum tunneling alone [Fig. 3(b)]. A factor of $10^{4}$ can be rapidly gleaned from the time axis when comparing the time dependence of the two curves.

\section{EXPERIMENTAL METHODS AND SAMPLE SYSTEMS}

There are several experimental techniques typically used for the preparation and investigation of spin chains on solid-state substrates depending on the nature of the spin chain and the properties to study. Traditional methods are measurements over ensembles of many spin chains and are thus averaging techniques such as, e.g., angle-resolved photoemission spectroscopy, magnetic susceptibility, calorimetry, electron-spin resonance, and neutron scattering. The advent of scanning probe techniques has permitted us access to each individual atom in a single-spin chain. This gives rise to new possibilities such as studying local properties by carefully positioning the scanning tunneling tip within the spin chain, or studying chains as a function of the number of atoms, their nature, and other parameters. This section is devoted to a brief description of the methods that can be used to study individual chains regarding their magnetic properties, their preparation, and the nature of the holding substrate.

\section{A. Experimental methods}

Here we review the methods based on scanning probe methods, particularly the STM. There are different STM measuring modes. The scanning modes typically give information on the topography of the studied objects. For spin chains they reveal important data on the atomic geometry and disposition with respect to the substrate. The typical imaging mode is the constant current mode where the set of tip heights over the sample are recorded while keeping the tunneling current constant. This very early measuring mode was shown to largely reproduce the spatial distribution of the constant local density of states (LDOS) of the substrate at its Fermi energy $E_{F}$ and at the tip's position (Tersoff and Hamann, 1985).

It was quickly realized that a second operation mode of the STM was to measure the differential conductance at a given tip location. Extending the interpretation of Tersoff and Hamann (1985) to finite bias $V$, this would yield precious information on the density of states at a given position, again the LDOS at the tip's location. Furthermore, advanced transport theory shows that in the presence of one conductance channel or under some simplifying assumptions about the tipsubstrate electronic coupling (Meir and Wingreen, 1992), the differential conductance is proportional to the many-body LDOS of the substrate, at $E_{F}+e V$. Measuring the differential 
conductance is tantamount to measuring the many-body spectral properties of the substrate, ranging from any type of excitation to the Kondo effect and to the general orbital structure of the system. In summary, the differential conductance contains information about all degrees of freedom of the substrate that can interact with an injected electron.

The different ways to measure the differential conductance give rise to different experimental techniques that we briefly review now.

Scanning tunneling spectroscopy.-In general, measuring the differential conductance at a given bias $V$ and tip position is the spectroscopic mode named scanning tunnel spectroscopy (STS). As we have just seen, it provides information about the spin-averaged electronic properties of a sample, and by using a magnetic tip also spin resolution is achieved (see later). Using the scanning capabilities of the STM, maps of differential conductance can be produced at different bias. When an interesting energy $E$ is identified spatially resolved $d I / d V$ maps at only the according bias voltage $V=E / e$ can be performed to reduce the measurement time.

Inelastic electron tunneling spectroscopy.-The main experimental difference with the previous spectroscopic mode, the STS, is the bias resolution that permits us to obtain a direct measurement of inelastic excitations. In order to increase the signal-to-noise ratio compared to numerically derived $d I / d V$, spectra lock-in techniques are applied. The modulation should be high enough to significantly reduce the $1 / f$ noise, but low enough to be still in the bandwidth of the amplifier. The modulation bias also reduces the noise at the expense of broadening the spectral features.

This measuring mode is usually known as inelastic electron tunneling spectroscopy (IETS). Vibrational modes ranging from a few to hundreds of meV have been detected with IETS (Stipe, Rezaei, and Ho, 1998; Ho, 2002; Komeda, 2005; Gawronski, Mehlhorn, and Morgenstern, 2008; Morgenstern, Lorente, and Rieder, 2013). This was a very exciting development because IETS permitted a chemical identification of adsorbed species that is generally absent in the large-energy scale of STS.

Figures 4(a) and 4(b) show typical IETS measurements. When the bias matches an excitation energy $V_{\mathrm{exc}}=E_{\mathrm{exc}} / e$, the tunneling electron can yield part of its energy and end up in a different state. The effective number of final states for tunneling suddenly increases at the threshold $V_{\text {exc }}=E_{\text {exc }} / e$. As a consequence, the tunneling current changes its slope, Fig. 4(a), which is more clearly seen in its derivative $d I / d V$ or differential conductance, Fig. 4(b). The steep increase at threshold and the electron-hole symmetry of the IETS signal are the hallmarks that the spectral features in the differential conductance correspond to an excitation.

These properties are common to any kind of excitation that can be induced by tunneling electrons. Spin can flip under a tunneling electron, giving rise to magnetic excitations that can be detected in the same way (Heinrich et al., 2004). This is of great value in the study of spin chains because it furnishes a characterization of the spin chain. Typical spin-flip excitations are in the meV range, where they can coexist with acoustic phonons that are difficult to excite by tunneling electrons (Gawronski, Mehlhorn, and Morgenstern, 2008). Contrary to phonons, spin-flip excitations are very easy to excite. A simple
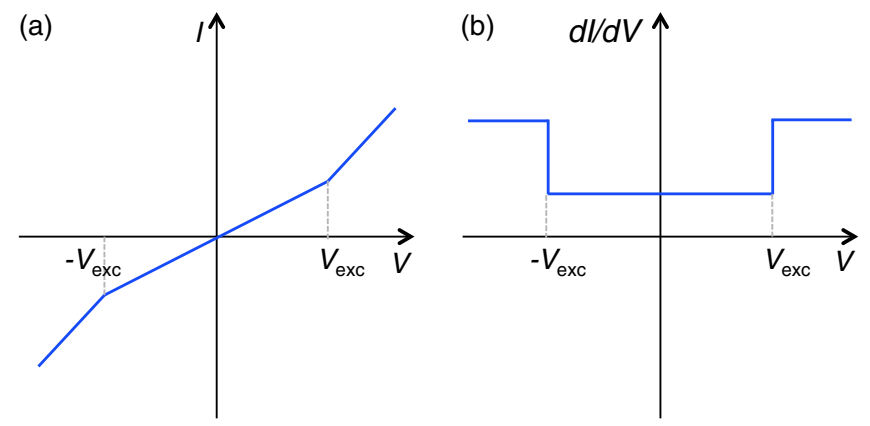

FIG. 4. (a) Current vs voltage spectrum with an additional tunnel channel at a threshold voltage $\left|V_{\text {exc }}\right|$ due to an inelastic excitation. (b) Inelastic excitations are typically studied measuring the differential conductance and then appear as symmetric steps at $-V_{\text {exc }}$ and $V_{\text {exc }}$ around the Fermi energy.

picture relates the change in conductance over the excitation threshold with the fraction of tunneling electrons that actually induce the excitation (Lorente, Rurali, and Tang, 2005). While vibrational excitation yields excitations in the range of $10 \%$, magnetic excitations easily exceed $100 \%$ (Lorente and Gauyacq, 2009).

Recently, it was shown that IETS can also detect orbital excitations. In this case, the symmetric signature of excitations in the IETS is also lifted because it depends on the occupancy of the orbitals (Kügel et al., 2018).

Spin-dependent tunnel processes.-When a magnetic tip is used, the tunneling current can be spin polarized (SP). This has implications for both the elastic as well as the inelastic contribution to the tunnel current (Bode, 2003; Wiesendanger, 2009; Loth, von Bergmann et al., 2010; Loth, Lutz, and Heinrich, 2010). For a static magnetization of a sample the spin polarization of the tunnel current and the differential conductance scales with the projection of sample onto tip magnetization, i.e., a tip magnetized along its axis is sensitive to the out-of-plane component of the sample magnetization, whereas a tip magnetization parallel to the surface plane detects in-plane magnetization components of the sample. These so-called spin-resolved STM (SP-STM) and spinresolved STS (SP-STS) modes allow access to the spatially resolved magnetic properties of magnetic atoms, nanostructures, or surfaces down to the atomic scale (Bode, 2003; Wiesendanger, 2009; Wiebe, Zhou, and Wiesendanger, 2011; von Bergmann et al., 2014).

As we saw for IETS, when the tunneling electron can induce a spin-flip excitation in the tunnel junction the spin polarization of a tunnel current leads to a preferred direction of excitation, i.e., the minority spin channel of the tip can flip a spin in one direction and the majority electrons flip it in the opposite direction. This leads to the lifting of electron-hole symmetry by having different amplitudes of the inelastic excitation steps at positive and negative bias in the differential conductance. The asymmetry scales with the spin polarization of the tunnel current for low tunneling rates. An additional source of bias asymmetry comes from the spin-conserving potential scattering that leads to interference with the spin-flip contribution. At higher tunneling rates, spin pumping can occur, because multiple subsequent excitations survive before deexcitation. This drives the system out of equilibrium with 
sizable bias asymmetries (Loth, von Bergmann et al., 2010; Loth, Lutz, and Heinrich, 2010).

Pump-probe techniques. - The dynamical evolution of spin excitations can be observed by using the previous pumping process. This has grown to become an STM-based electronic pump-probe technique. One of the first applications was to measure the spin relaxation time of a $\mathrm{Fe}-\mathrm{Cu}$ coupled dimer on the $\mathrm{Cu}_{2} \mathrm{~N}$ surface (Loth, Etzkorn et al., 2010).

The technique uses a series of electronic pump and probe pulses that are generated and sent to the STM; see Fig. 5(a). Once the electronic pump pulse is sent to the adsorbates on the surface, the spins of the system excite and relax over time. The voltage of the pump pulse has to be larger than the excitation energy to excite the spin from the ground state to an excited state by inelastic scattering of tunneling electrons. A probe pulse of smaller voltage is sent to measure the state of the spin. This is achieved by magnetically polarizing the STM tip. By sending the probe pulse at different time delays $(\Delta t)$, information on the time dependence of the population of the levels can be obtained by measuring the evolution of conductance with $\Delta t$; see Fig. 5(b). The conductance behaves exponentially with $\Delta t$, a characteristic time constant given by the spin relaxation time $\left(T_{1}\right)$. The working principle of this technique is tunneling magnetoresistance. After the spin is excited by a pump pulse, the spin relaxes and goes to the ground state. Using a spinpolarized tip, depending on the orientation of the adsorbed spin at certain time delay, the conductance will change with the characteristic $T_{1}$ time constant.

Using this technique, the spin relaxation time of $\mathrm{Fe}$ spin chains has been measured as a magnetic tip was being approached (Yan et al., 2015). The exchange field of the tip changed the state mixing of the spin chain, and this had a measurable effect on the lifetime of the spin-chain excitations.

\section{B. Preparation of chains on surfaces}

There are two possibilities to obtain well-defined chains on surfaces: self-organization or atom manipulation. A spontaneous

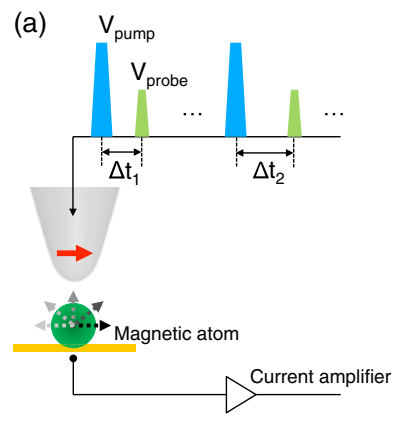

(b)

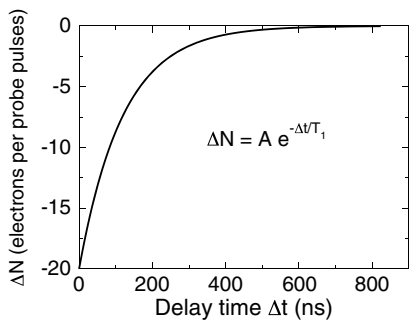

FIG. 5. Simplified diagram of the electronic pump-probe technique. (a) First, the pump pulse excites the spin states of the adsorbed magnetic atom and second, the probe pulse at certain time delay $\left(\Delta t_{1}\right)$ detects the status of the spin by spinpolarized tunneling. By varying the time delay $(\Delta t)$ and sending repeated sets of pump-probe pulses, (b) the conductance as a function of time is obtained, which gives information of the spin relaxation time $\left(T_{1}\right)$. In the present example the initial number of collected electrons is -20 and the relaxation time is $120 \mathrm{~ns}$. Adapted from Loth, Etzkorn et al., 2010. formation of spin chains can be realized on uniaxial surfaces, taking advantage of surface thermodynamics (Himpsel et al., 1998; Gambardella et al., 2000, 2002). In particular, metallic substrates allow for the required atom diffusion for selforganized growth and it was shown that tens of nanometer-long one-dimensional spin chains can be reproducibly achieved. Atom manipulation with the tip of an STM was first demonstrated for Xe atoms on a Ni(110) surface (Eigler and Schweizer, 1990). In both lateral and vertical manipulation modes the force between the tip and a single atom adsorbed on a surface is exploited to reproducibly displace the single atom (Bartels, Meyer, and Rieder, 1997), making it possible to build nanostructures atom by atom (Lorente, Rurali, and Tang, 2005; Morgenstern, Lorente, and Rieder, 2013). Typically the potential landscape for diffusion on metal surfaces is smooth enough to allow for lateral manipulation of adatoms, whereas surfaces that form covalent bonds with the adatom such as a $\mathrm{Cu}_{2} \mathrm{~N}$ layer grown on $\mathrm{Cu}(100)$, that has been the substrate for various magnetic chains as discussed later, requires the pickup and dropoff of single atoms with the tip, i.e., vertical manipulation.

\section{Spin chains and their holding substrates}

In this Colloquium, we focus on atomic spin chains on a surface. In this scenario, the influences of the substrate on the (magnetic) properties of the spin chain become an important consideration, and one of the main factors is the coupling strength to the substrate's electron bath. When a spin chain is only weakly coupled to an electron bath, as for lightly doped semiconductor substrates, the low electron concentration impedes electronic excitations of the low-energy magnetic states of a spin chain. Only phonons are available for damping and they are not very efficient because (i) they need a large spin-orbit interaction to couple spin and atomic-position degrees of freedom and (ii) the number of available phonons is very limited at low temperatures. However, in lightly doped semiconductors spin excitations are difficult to detect experimentally, due to practicalities related to detecting changes in conductance when the applied bias is large enough to overcome the electronic band gap (Khajetoorians et al., 2010). To circumvent this, a metal substrate passivated with a semiconducting or insulating film can be used as a substrate for magnetic chains. In such a sample, the passivation of the metal substrate reduces the coupling with the electron bath, hence increasing the lifetime of the intrinsic spin-chain excitations while permitting good conductance conditions to resolve the electronic current from the STM tip. A particularly successful substrate for the construction of extended spin structures has been the case of a monolayer of $\mathrm{Cu}_{2} \mathrm{~N}$ grown on $\mathrm{Cu}(100)$ (Hirjibehedin, Lutz, and Heinrich, 2006; Hirjibehedin et al., 2007; Otte et al., 2008; Loth, von Bergmann et al., 2010; Spinelli et al., 2014; Bryant et al., 2015; Choi, Robles, Gauyacq, Rubio-Verdú et al., 2016; Choi, Robles, Gauyacq, Ternes et al., 2016); see Sec. IV.

On the other hand, in order to strongly couple the magnetic chain to an electron bath, the chain atoms can be adsorbed directly to a metal substrate, which efficiently couples their orbitals to the delocalized electrons of the substrate. In this case, any excitation of the system is damped relatively quickly due to the enhanced coupling. The damping occurs via 
efficient electron-hole excitations, which dominate over other deexcitation channels even at very low temperatures. When an atomic system of spins is in contact with a metal surface, the magnetic spectra become broadened by this mechanism. This leads to broad features in the differential conductance spectra which, nevertheless, can still be detected with the STM (Balashov et al., 2009; Schuh et al., 2010; Chilian et al., 2011; Khajetoorians, Lounis et al., 2011; Khajetoorians, Schlenk et al., 2013). At the same time the strong hybridization with the substrate can lead to induced magnetic moments in the substrate atoms, and often the magnetic properties can be understood within the classical limit. In addition to the investigation of dense and dilute chains on such normal metal surfaces, there has recently been increased interest in the properties of magnetic chains on superconductors; see Sec. V.B.

\section{SPIN CHAINS DECOUPLED FROM THE SUBSTRATE'S ELECTRON BATH}

\section{A. Passivated metal substrates}

The first demonstration of spin excitations on a passivated metal substrate was performed by Heinrich et al. (2004). They used $\mathrm{Al}_{2} \mathrm{O}_{3}$ islands grown on $\mathrm{NiAl}$ to deposit a small number of $\mathrm{Mn}$ atoms where they performed conductance measurements as a function of bias at low temperature $(0.6 \mathrm{~K})$ and with magnetic fields as high as $7 \mathrm{~T}$. Other, more recent experiments involve single atoms and small multiatom structures on $\mathrm{MgO}$ on $\operatorname{Ag}(100)$ (Rau et al., 2014; Baumann et al., 2015; Baumann, Paul et al., 2015; Natterer et al., 2017), providing the opportunity to tune the coupling strength to the substrate by varying the number of $\mathrm{MgO}$ layers. A particularly successful substrate for the construction of extended spin structures has been the case of a monolayer of $\mathrm{Cu}_{2} \mathrm{~N}$ grown on $\mathrm{Cu}(100)$ (Hirjibehedin, Lutz, and Heinrich, 2006; Hirjibehedin et al., 2007; Otte et al., 2008; Loth, von Bergmann et al., 2010; Spinelli et al., 2014; Bryant et al., 2015; Choi, Robles, Gauyacq, Rubio-Verdú et al., 2016; Choi, Robles, Gauyacq, Ternes et al., 2016), which is the main focus of this section.

Bulk copper nitride is an insulator with a gap of above $4 \mathrm{eV}$. A single atomic layer does not form a complete insulator and only partially decouples the spin from the copper metal substrate. These conditions turn out to be ideal for IETS experiments. In addition to acting as a decoupling layer, the $\mathrm{Cu}_{2} \mathrm{~N}$ surface forms a good template grid for assembling spin chains. The $\mathrm{N}$ atoms are bonded covalently to the $\mathrm{Cu}$ atoms, making the $\mathrm{Cu}_{2} \mathrm{~N}$ layer essentially a two-dimensional molecule with square symmetry (Hirjibehedin et al., 2007). When a transition metal atom, such as $\mathrm{Co}, \mathrm{Fe}$, or $\mathrm{Mn}$, is positioned on top of the layer, it is incorporated into that molecule. As such, manipulation of adatoms on top of $\mathrm{Cu}_{2} \mathrm{~N}$ can be seen as the construction of a two-dimensional magnetic molecule with spin centers at predeterminable locations.

Density functional theory (DFT) calculations (Rudenko et al., 2009; Urdaniz, Barral, and Llois, 2012; Choi, Robles, Gauyacq, Rubio-Verdú et al., 2016) show that the $\mathrm{Cu}_{2} \mathrm{~N}$ monolayer is profoundly modified when a magnetic atom is placed directly over a $\mathrm{Cu}$ atom - the typical binding site for transition metal atoms. The $\mathrm{Cu}$ atom underneath the magnetic atom is pushed into the substrate while the two neighboring $\mathrm{N}$ atoms are pulled upward into the chain. As a consequence, we can view a spin chain built on $\mathrm{Cu}_{2} \mathrm{~N}$ as an ensemble of alternating TM atoms and $\mathrm{N}$ atoms.

The crystal field due to the nitrogen network can provide an anisotropic environment with clear preferred magnetization axes for the spins (Hirjibehedin et al., 2007). For the magnetocrystalline anisotropy encountered on the $\mathrm{Cu}_{2} \mathrm{~N}$ surface, typically the second order form of Eq. (5) involving a uniaxial parameter $D$ and a transverse term $E$ suffices. A study by Bryant et al. (2013) showed that these phenomenological parameters can be understood readily in terms of the angle between the two nitrogen bonds pointing away from the magnetic atom. The closer this angle is to $180^{\circ}$, the larger the ratio $D / E$. The exact geometry of atoms incorporated into the network will be discussed further later.

The molecular nitrogen network is also responsible, at least in part, for mediating spin-spin coupling over distances spanning several unit cells (Otte et al., 2008). By placing pairs of magnetic atoms near each other on the network, depending on their relative positioning different coupling signs and strengths are found with values of the Heisenberg parameter $J$ ranging from $J \sim+2 \mathrm{meV}$ (antiferromagnetic) to $J \sim$ $-1 \mathrm{meV}$ (ferromagnetic) (Spinelli, Rebergen, and Otte, 2015). While the exact physical mechanism underlying the spinspin coupling remains to be studied further, it is believed to be a combination of superexchange mediated by the nitrogen network and RKKY coupling (Yosida, 2001) mediated through the metal underneath. In general, it is found that the coupling strength decreases rapidly with the number of nitrogen bonds separating the atoms.

The first spin chains on $\mathrm{Cu}_{2} \mathrm{~N}$ were built by Hirjibehedin, Lutz, and Heinrich (2006). They showed that $\mathrm{Mn}_{n}$ chains could be built with $n=2, \ldots, 10$ by using a vertical atom manipulation technique; see Fig. 6(a). The atoms were placed one unit cell apart, separated by a single N atom; see Fig. 6(b). IETS showed clear and distinct behavior depending on the parity of $n$. For chains with an even number of Mn atoms, clear excitation thresholds appeared at several meV that reduced in energy as the number of $\mathrm{Mn}$ atoms increased. Odd-numbered chains, on the other hand, displayed a small bias featured reminiscent of the small magnetic anisotropy of a single Mn atom; see Fig. 6(c). These spectra were readily explained in terms of an isotropic Heisenberg Hamiltonian with antiferromagnetic coupling between the spins in the chains. In contrast to other spin chains discussed later, in the case of these close-spaced Mn chains spectroscopy was found to be the same regardless of the position of the tip on the chain. As such, the chains could be viewed as a single magnetic entity, using the giant spin approximation. For even-numbered chains, having zero total spin in the ground state, the observed excitation corresponds to a singlet-triplet excitation. Oddnumbered chains, on the other hand, have a net spin of 5/2. Similar to the single Mn atom, their spectra display only a small-bias dip. Indeed, the exchange interaction obtained in this way matched the computed exchange interactions for the same system (Rudenko et al., 2009; Urdaniz, Barral, and Llois, 2012). 
(a)

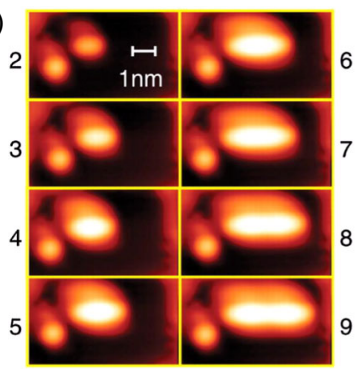

(b)

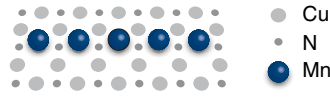

(c)

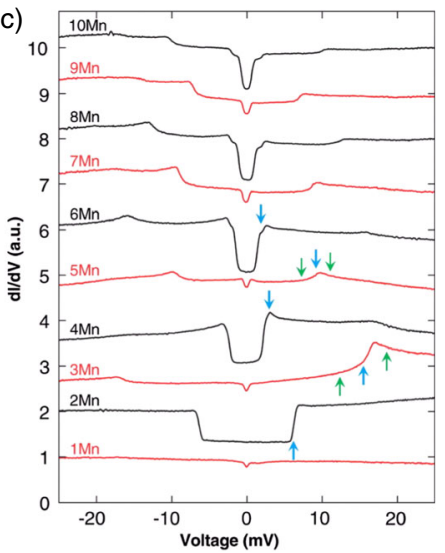

FIG. 6. Constant current images (a) of the created $\mathrm{Mn}_{n}$ chains with $n=2, \ldots, 9$ on $\mathrm{Cu}_{2} \mathrm{~N} / \mathrm{Cu}(100)$. (b) Atomic scheme where the $\mathrm{Mn}$ atoms are depicted by blue (dark gray) balls, $\mathrm{N}$ atoms are represented by small circles, and $\mathrm{Cu}$ atoms by large circles. (c) Differential conductance over atomically manipulated $\mathrm{Mn}_{n}$ chains with $n=1, \ldots, 10$ (b). Depending on the number of Mn atoms, the behavior is different. For odd number, it shows a small bias feature with the spin changing excitation energy steps while for even number, it gives only the spin excitation energy steps. The lowest spin changing excitations are marked by blue arrows. Adapted from Hirjibehedin, Lutz, and Heinrich, 2006.

Follow-up work focused predominantly on Fe chains. Loth et al. (2012) showed that for chains with an interatomic spacing of two unit cells (which can essentially be seen as repetitions of a $\mathrm{Fe}-\mathrm{N}-\mathrm{Cu}-\mathrm{N}$ unit cell), the magnetic ordering was still antiferromagnetic. But in contrast to the earlier closed-spaced Mn chains, spectroscopy performed on each of the atoms in the double-spaced Fe chains showed different excitation intensity, justifying a description in terms of weakly coupled local magnetic moments. Spin-polarized measurements indicated that the two lowest-energy states of the Fe spin chains are Néel states that correspond to a classical arrangement of opposing spins. Under the influence of either tunneling current or temperature, switching between the two possible Néel states could be induced. The study of the efficiency of the switching as a function of applied bias permitted them to determine a threshold and hence identify an indirect mechanism for switching. An impinging electron would excite the spin chain into an excited state followed by a decay into the other Néel state. Calculations proved that collective excitations were at play (Gauyacq et al., 2013). The threshold was determined by the excitation of the lowestenergy spin wave of the chain. As the energy of the tunneling electron increased, more excitations of the chain could be excited improving the switching mechanism to the point that a $50 \%-50 \%$ deexcitation probability into either of the two Néel states was reached (Gauyacq et al., 2013).

Advanced measurements on these spin chains showed that the combination of IETS and the sudden variation in spinpolarized current due to the change in state population near the excitation threshold could lead to peculiar spectroscopic features including negative differential conductance (RolfPissarczyk et al., 2017). In addition, it was shown that the chains could be flipped as well due to the effect of exchange bias with the magnetized STM tip, provided that the tip was

brought in sufficiently close proximity to the structure (Yan et al., 2015).

An experimental study focused on the collective excitations that are populated during the switching process was provided by Spinelli et al. (2014). Here they studied chains of Fe atoms that, due to a different orientation of the chains on $\mathrm{Cu}_{2} \mathrm{~N}$, were coupled ferromagnetically; see Fig. 7(a). The resulting two metastable states were states where all the spins were parallel to each other and pointing in one of the two opposing directions along the easy axis. Also here, telegraphic switching between the two metastable states was observed; see Fig. 7(b). In particular, the switching was found to be current induced and dependent on the location of the STM tip over the chain. IETS measurements performed on each of the atoms in the chain revealed that the lowest-energy excitations were of a spin-wave nature: a clear nodal structure was observed, with the number of nodes increasing for higher energy modes; see Fig. 7(c). Rate equation calculations indicated that the lowest-energy transitions from one metastable state to the other passed through a sequence of these spin waves states, followed by domain-wall states sweeping the domain from one end of the chain to the other (Spinelli et al., 2014).

Spin chains made of Co atoms have shown a very different behavior (Bryant et al., 2015). Intriguingly, clear IETS measurements could be performed on only the edges of the spin chains, while no signal was recorded over the bulk sites. The explanation of this peculiar behavior lies in the actual geometry of the chain: the edge atoms have a finite $\mathrm{N}-\mathrm{Co}-\mathrm{N}$

(a)
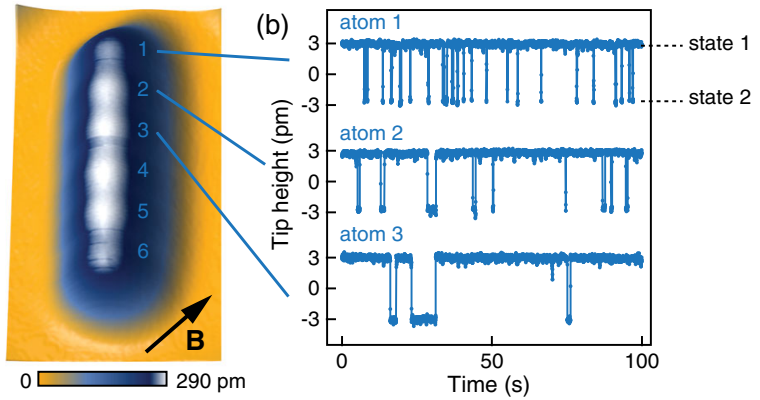

(c)

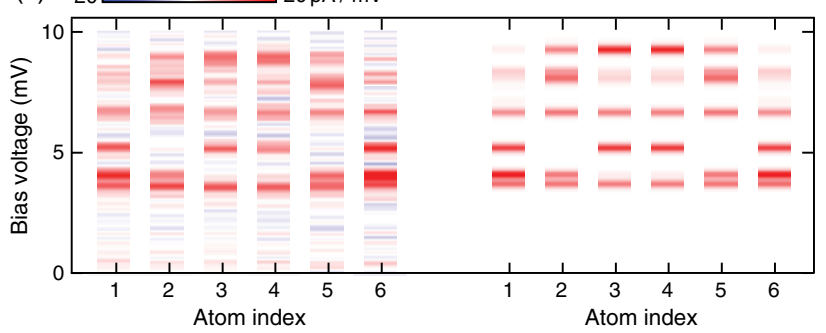

FIG. 7. Detection of spin waves in a ferromagnetic chain. (a) STM topography of a ferromagnetic six-atom Fe chain on $\mathrm{Cu}_{2} \mathrm{~N} / \mathrm{Cu}(100)$. (b) Telegraph noise measured using spinpolarized STM on the first three atoms of the chain. Switching is observed between two metastable states. The switching rate decreases as the tip is moved toward the center of the chain. (c) Left: IETS spectra taken on each of the atoms in the chain. Spin-wave states are observed with recognizable nodal structure at $\sim 3.5, \sim 4.0$, and $\sim 5.5 \mathrm{mV}$. Right: Corresponding theory obtained from diagonalization of the spin Hamiltonian. Adapted from Spinelli et al., 2014. 
angle, whereas for the atoms away from the edges the N-Co bonds were almost collinear. This leads to an electronic structure where there is no overlap between the tip apex and the $d$ orbitals of the bulk Co atoms. As a result, interaction of the tunneling electrons with the local spins of the chain is avoided, preventing spin excitations from occurring (Bryant et al., 2015).

Recently, Co chains of a different configuration were shown to be a useful platform for making experimental realizations of model spin Hamiltonians (Toskovic et al., 2016). While Co atoms in principle have a spin magnitude $S=3 / 2$, here they demonstrated that an effective spin-1/2 chain can be engineered by making use of magnetocrystalline anisotropy. Co atoms on $\mathrm{Cu}_{2} \mathrm{~N}$ are found to experience hard axis anisotropy as a result of which the $m= \pm 1 / 2$ Kramers doublet is split off approximately $6 \mathrm{meV}$ below the $m= \pm 3 / 2$ doublet; see Fig. 8(a). By designing the antiferromagnetically coupled chain such that the coupling strength $J$ between the spins is much smaller than $6 \mathrm{meV}$, an effective spin-1/2 chain with anisotropic $X X Z$ coupling is formed (Toskovic et al., 2016); see Fig. 8(b). The model $X X Z$ Heisenberg Hamiltonian is known for a critical point at a certain value of the transverse magnetic field, beyond which the chain becomes paramagnetic. Before reaching this critical point, the system is characterized by a ground state doublet which is topologically separated by an excitation gap from the continuum of states (Dmitriev, Krivnov, and Ovchinnikov, 2002). Local spectroscopy measurements on the Co chains as a function of the
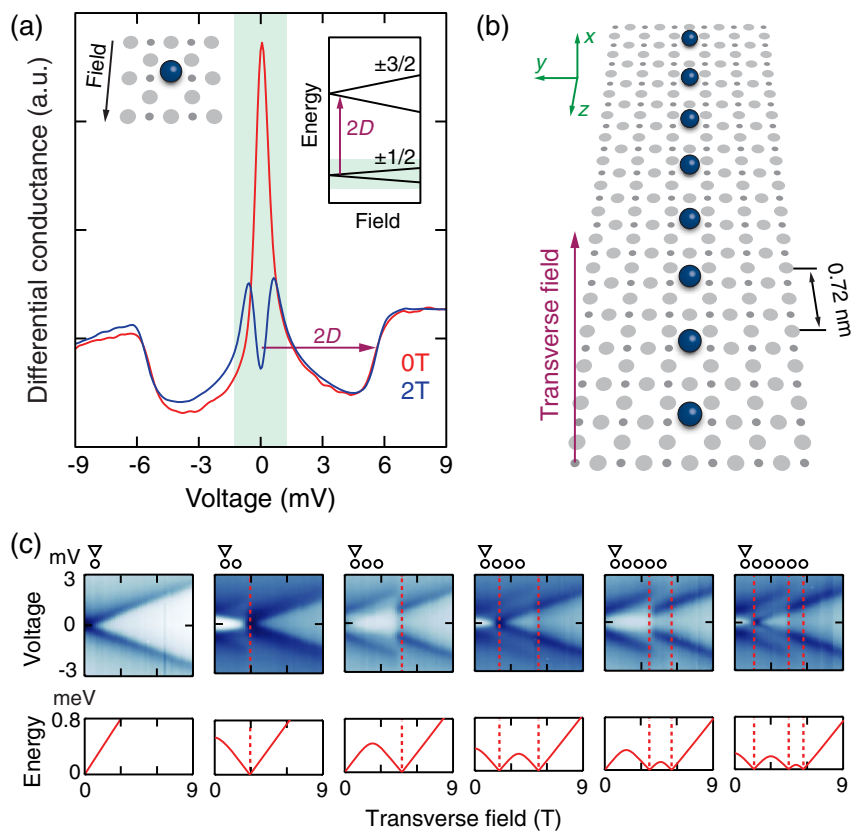

FIG. 8. Using an atomic spin chain for quantum simulation. (a) IETS spectrum taken on a single $\mathrm{Co}$ atom on $\mathrm{Cu}_{2} \mathrm{~N} / \mathrm{Cu}(100)$, indicating a split between the $\pm 1 / 2$ and $\pm 3 / 2$ doublets. (b) Atomic design of an $X X Z$ Heisenberg chain in transverse field using Co atoms. (c) Top: IETS performed on the first atom of chains up to length 6 for transverse magnetic fields up to $9 \mathrm{~T}$. Transitions in the ground state are observed leading up to the critical point near $6 \mathrm{~T}$, which coincide with theoretically predicted ground state changes (bottom). Adapted from Toskovic et al., 2016. transverse magnetic field revealed these two states and their interplay in the region leading up to the critical point; see Fig. 8(c).

\section{B. The impurity problem and its extension to spin chains}

An interesting twist to these results comes when heterogeneous spin chains are used. Indeed, if we can consider a spin chain as a single magnetic object in contact with a reservoir of electrons (the substrate), a Kondo effect due to the collective behavior of the spin chain can take place. The Kondo effect (Hewson, 1993) is due to the electronic correlations caused by spin-flip scattering off a magnetic impurity. The magnetic impurity has to present a twofold degenerate ground state in the absence of spin flips that can be switched one into the other by zero-energy spin flips (Choi and Lorente, 2018). In order to achieve this in a spin chain, all spins need to be strongly entangled.

In the previous section, we showed the case of Mn chains (Hirjibehedin, Lutz, and Heinrich, 2006; Choi, Robles, Gauyacq, Rubio-Verdú et al., 2016). Chains with an odd number of $\mathrm{Mn}$ atoms have a 5/2 ground state that cannot be connected via $\Delta S_{z}= \pm 1$ spin flip. Hence, no Kondo effect takes place. Even-numbered antiferromagnetic chains are singlets so they have no degeneracy. As a consequence, no Kondo effect is revealed in the $d I / d V$ spectra of these chains. Other antiferromagnetically coupled chains such as $\mathrm{Fe}_{n}$ (Loth et al., 2012; Spinelli, Rebergen, and Otte, 2015) and $\mathrm{Co}_{n}$ (Bryant et al., 2015) show no degenerate ground state either preventing the formation of Kondo correlations. It seems difficult to have a spin chain with a degenerate ground state that can be connected via spin flips. The solution was found by Choi, Robles et al. (2017) by building heterogeneous chains with two ingredients: (i) a twofold degenerate spin ground state in the absence of spin flips, and (ii) strong entanglement such that a single spin flip from a substrate electron can reverse the ground state.

Choi, Robles et al. (2017) built $\mathrm{Mn}_{n}$ chains where they added a terminal $\mathrm{Fe}$ atom. The newly created $\mathrm{FeMn}_{n}$ chains were in principle $S=1 / 2$ systems for an odd number of $\mathrm{Mn}$ atoms (odd $n$ ) since all atoms couple antiferromagnetically along the nitrogen rows of the $\mathrm{Cu}_{2} \mathrm{~N} / \mathrm{Cu}(100)$ substrate. The same could also be said of $\mathrm{Fe}_{n} \mathrm{Mn}$ chains, since again, for odd $n$ the sum of antiferromagnetically aligned magnetic moments leads to $1 / 2$. However, the experiment showed that these two systems behave very differently. In the case of $n=3$, the $\mathrm{FeMn}_{3}$ chain displayed a zero-bias anomaly that was shown to be a Kondo peak while the $\mathrm{Fe}_{3} \mathrm{Mn}$ chain displayed no Kondo peak (Choi, Robles et al., 2017). They realized that besides the exchange interaction controlling the spin-spin coupling along the chain, the magnetic anisotropy of each atomic spin was important. Indeed, Fe presents a large axial magnetic anisotropy as compared to Mn. Assuming similar exchange couplings, the Fe-rich chains will tend to align their spins more than the Mn-rich chains. Thus, a $\mathrm{Fe}_{n} \mathrm{Mn}$ chain will have a larger number of spins that prefer to align along the atomic spin axis, leading to an Ising-like spin system and hence to a system with reduced entanglement. Flipping the full spin of the $\mathrm{Fe}_{n} \mathrm{Mn}$ chain via a substrate spin flip becomes difficult. However, the magnetic anisotropy of $\mathrm{FeMn}_{n}$ is very reduced 
and the ground state strongly resembles the one of an antiferromagnetic Heisenberg chain strongly entangled. As a consequence a single spin flip from the substrate has a larger probability of flipping the full spin of the chain leading to the Kondo phenomenon.

Figure 9 summarizes the behavior of $\mathrm{FeMn}_{n}$ spin chains ( $n=1-9$ ) on $\mathrm{Cu}_{2} \mathrm{~N} / \mathrm{Cu}(100)$. Similarly to the $\mathrm{Mn}_{n}$ spin chain, the atomic structure of the chain includes strong relaxation of the surface as shown in the results of DFT calculations plotted in Fig. 9(a). There the incorporation of $\mathrm{N}$ atoms into the chain is evident as well as the reorganization of the nearest $\mathrm{Cu}$ atoms. Despite the chain being mostly a $\mathrm{Mn}_{n}$ spin chain, the addition of an extra Fe changes the spectral features. In Fig. 9(b) a clear Kondo feature is localized on the edge of the chain that does not contain the Fe atom despite the fact that without Fe, there is no Kondo peak. This behavior can be explained only if indeed the chain is reacting like a single object allowed by the entanglement of spins. That the peak at zero bias is indeed a Kondo peak is shown in Fig. 9(c), where

(a)
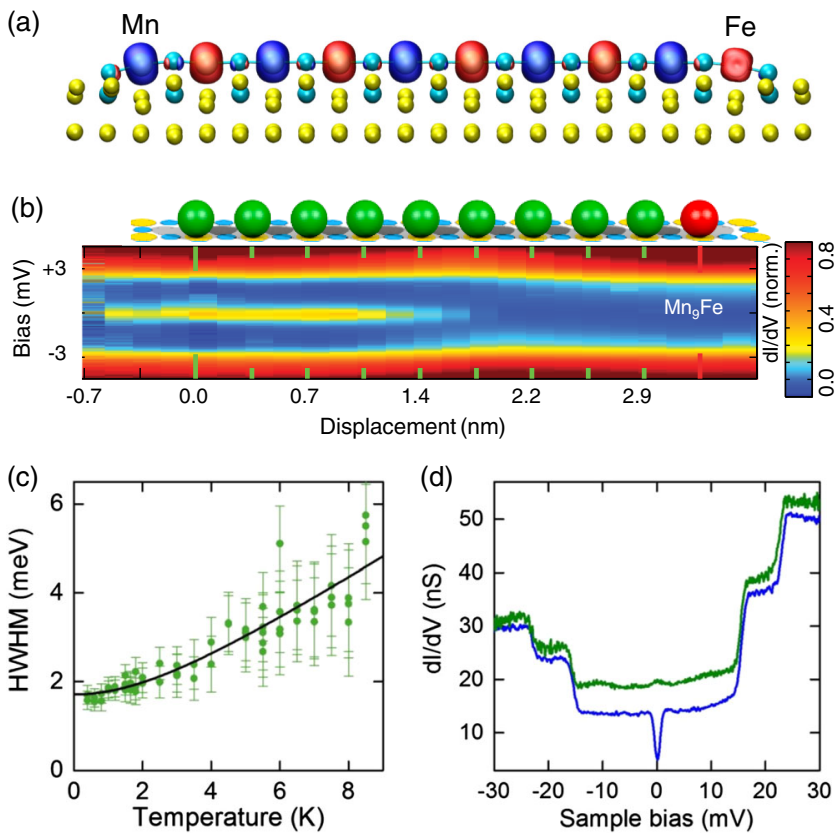

FIG. 9. Heterogeneous $\mathrm{FeMn}_{n}$ spin chains $(n=1-9)$, on $\mathrm{Cu}_{2} \mathrm{~N} / \mathrm{Cu}(100)$. (a) Density difference between spin-up (blue, dark gray) and spin-down (red, light gray) states over a $\mathrm{Mn}_{9} \mathrm{Fe}$ chain. Copper atoms (yellow, light gray) and nitrogen atoms (cyan, dark gray). (b) Differential conductance $(d I / d V)$ map along the $\mathrm{Mn}_{9} \mathrm{Fe}$ chain. $d I / d V$ signals plotted as a function of sample bias (V) and displacement (nm). Mn (green, dark gray) and Fe (red, light gray) atoms are visualized to show where they sit. (c) Half width at half maximum (HWHM) (meV) of the zero-bias anomaly for the $\mathrm{MnFe}$ dimer plotted as a function of temperature. The black line is a fit to the Kondo peak as a function of temperature. (d) The differential conductance as a function of applied bias for two different magnetic fields in the $\mathrm{MnFe}$ dimer. The green (upper) curve corresponds to no magnetic field and the blue one to a $B=5 \mathrm{~T}$ field applied perpendicular to the surface. The measuring temperature is $0.5 \mathrm{~K}$. The green line (upper line) is vertically shifted by $5 \mathrm{nS}$ for clarity. Adapted from Choi, Robles et al., 2017. the behavior of the zero-bias peak with temperature follows the trend of a Kondo peak. The data are fitted by a function $\Gamma_{K}(T)$ that takes into account the thermal broadening of the resonance and the tip's Fermi function (Nagaoka et al., 2002; Ternes, Heinrich, and Schneider, 2009):

$$
\Gamma_{K}(T)=\frac{1}{2} \sqrt{\left(2 \Gamma_{K}^{0}\right)^{2}+\left(3.5 k_{B} T\right)^{2}+\left(\alpha k_{B} T\right)^{2}} .
$$

The coefficient $\alpha$ reflects how close to a Fermi-liquid solution the Kondo system is (Nagaoka et al., 2002; Ternes, Heinrich, and Schneider, 2009; Ternes, 2015). The fit of Fig. 9(c) reveals a $T=0 \mathrm{~K}$ Kondo width $\Gamma_{K}^{0}$ of $1.68 \mathrm{meV}$ and a $\alpha$ value of 11.1 . These values agree with the behavior of a Kondo system. Moreover, the large value for $\alpha$ points at the behavior of a Kondo system in the weak coupling regime (Y.-h. Zhang et al., 2013). Further evidence can be found in Fig. 9(d) where the effect of the magnetic field splits the Kondo peak as expected. When a magnetic field of $5 \mathrm{~T}$ is perpendicular to the sample, the $S=1 / 2$-like ground state splits and the elastic spin flips giving rise to the Kondo peak cannot be produced anymore. The Kondo peak disappears and instead a clear inelastic spinflip signal develops as can be seen in the blue line of the graph.

\section{Semiconductor substrates}

Semiconducting substrates principally offer a large flexibility for the tuning of the properties of spin chains. The substrate electron density, and thereby the coupling of the chain spins to the electron bath, can be largely adjusted by the doping of the used semiconductor materials. Thereby, it is essentially possible to investigate the continuous transition from the decoupled spin case of the passivated surfaces to the strongly coupled case of the metallic substrate (Sec. V). The (110) surfaces of narrow gap III-V semiconductors, e.g., InAs and InSb, additionally feature the possibility to change the dimensionality of the electron bath between 3D, 2D, 1D, and even 0D, by surface doping, polar step edges, or using the STM-tip induced quantum dot (Meyer et al., 2003; Morgenstern, 2003; Wiebe et al., 2003; Hashimoto et al., 2008). Despite all these advantages, studies of spin chains on semiconductors are yet relatively sparse due to preparation and measurement difficulties.

For (110) surfaces, STM-tip based manipulation is rather uncontrolled because they form strong covalent bonds with metal adsorbates (Kitchen et al., 2006; Kitchen, 2006; Richardella, Kitchen, and Yazdani, 2009; Gohlke et al., 2013). Fölsch et al. (2009, 2014), Yang et al. (2012), and Pan et al. (2015) succeeded in the creation of individual chains of metal atoms on the (111) surface of molecular-beam epitaxy grown InAs by STM-based atom manipulation. STS of their electronic properties demonstrates the fascinating possibility to control the chain's and substrate's electronic properties down to the single atom limit. However, the spin properties of such chains have not yet been studied.

Self-organized growth of metal chains is limited to a few substrates (Matsui et al., 2007; Snijders and Weitering, 2010; van Houselt et al., 2013). STS investigations of the electronic properties of individual gold chains grown by self-assembly on stepped (Crain and Pierce, 2005) and flat (Do and Yeom, 
2015) Si substrates have been performed. They conclusively showed the 1D character of confined electron states in gold chains. Although the states in such chains have been predicted to be spin polarized (Erwin and Himpsel, 2010) investigations using SP-STS or IETS are, so far, lacking. Matsui et al. (2007) described room-temperature deposition of $\mathrm{Fe}$ on the (110) surface of InAs prepared by cleavage in ultrahigh vacuum. This procedure results in the self-assembly of sparse short chains with a maximum length of four to five atoms. The chain's electronic structure studied by STS revealed a strong dependence on the orientation of the chain with respect to the substrate orientation. But, also for this system, local investigations of the spin-related properties are, so far, lacking.

Finally, obtaining electrical signals of spin excitations of atoms on semiconductors is hampered by the gap in the density of states of the substrate that necessitates a relatively large stabilization bias for STS. Notwithstanding this difficulty, it has been possible to detect spin excitations of individual $\mathrm{Fe}$ atoms adsorbed to the (110) surface of InSb using IETS (Khajetoorians et al., 2010). In this work, the dilute surface electron doping via the $\mathrm{Fe}$ atoms acting as donors induces a $2 \mathrm{D}$ electron system at the surface which circumvents the problem opposed by the band gap. Most remarkably, the 2D electron system interacts with the spins of the Fe atoms in an interesting fashion. Namely, it has been shown that, in an applied magnetic field, the $S=1 \mathrm{Fe}$ spins acts as spin filters for the substrate electrons that tunnel between the spin-polarized Landau levels of the $2 \mathrm{D}$ electron system and the metal STM tip. This indicates a considerable exchange interaction between the electrons of the 2D system and the Fe spins. Unfortunately, so far, also for this promising sample system no chains were prepared and studied using IETS or SP-STS.

\section{SPIN CHAINS STRONGLY COUPLED TO THE SUBSTRATE'S ELECTRON BATH}

At low temperature, electronic excitations are the primary source of spin deexcitation and decoherence. We saw in the previous section how quantum properties of spin chains can be singled out and preserved by decoupling the atomic spins from the electronic degrees of freedom of the substrate. On the other hand, spin chains that are strongly coupled to substrates with ubiquitous electronic states are also of fundamental interest. In this case, the delocalized electronic states can efficiently mediate spin-spin long-range interactions (RKKY) including a considerable Dzyaloshinskii-Moriya contribution. This permits us to tailor the spin states by choosing the substrate material and the interatomic distances in the chain. Using heavy-element and/or superconducting metals as substrates, intriguing emergent properties like spin spirals or 1D topological superconductors can be realized, as we will review in the following.

\section{A. Spin chains on metallic substrates}

Chains by self-organization.-An early prominent example of the preparation and investigation of magnetic chains on metallic surfaces was Co chains grown by self-organization on a Pt surface (Gambardella et al., 2002). The substrate was a vicinal $\mathrm{Pt}(997)$ surface obtained by cutting a Pt crystal with a misalignment of $6.45^{\circ}$ relative to the (111) plane. In this case, rather narrow terraces with (111) orientation are formed and the deposited Co atoms tend to bind at the step edges, forming long one-dimensional Co chains; see Fig. 10(a). Using x-ray magnetic circular dichroism the experiments showed that, at low enough temperatures, these chains form a long-rangeordered ferromagnetic state due to a rather large magnetic anisotropy. Interestingly the easy axis is canted by $43^{\circ}$ with respect to the (111) surface normal toward the steps. Further physical insight can be obtained by studying the properties of single chains using SP-STM.

In addition to substrates with large miscut angles as for $\mathrm{Pt}$ (997), uniaxial surfaces can also be used as templates for the self-organized growth of chains. The growth of chains consisting of magnetic atoms has been demonstrated for the reconstructed $(5 \times 1)-\operatorname{Ir}(001)$ surface (Heinz and Hammer, 2009) [see atomically resolved STM image in Fig. 10(b)], and different kinds of biatomic chains have been studied regarding their magnetic properties (Menzel et al., 2012; Dupé et al., 2015). The magnetic atoms can adsorb on different adsorption sites leading to biatomic chains with different symmetry [Fig. 10(c)]: the atoms can sit in adjacent hollow sites
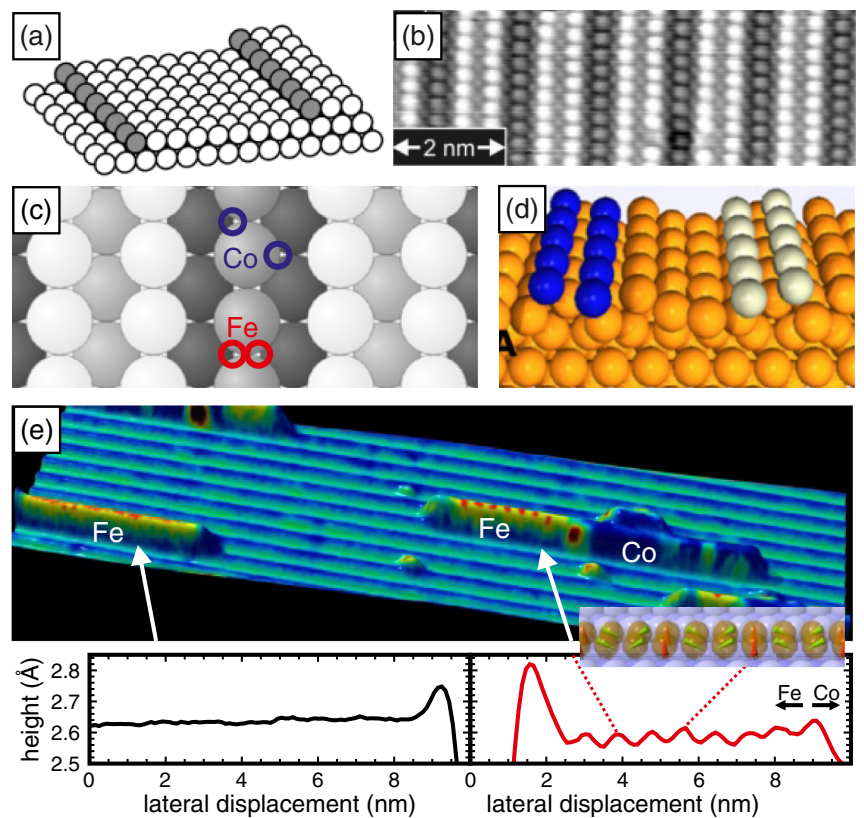

FIG. 10. Self-organized magnetic chains on metallic substrates. (a) Sketch of monatomic Co chains attached to the step edges of a $\mathrm{Pt}(997)$ single crystal as reported by Gambardella et al. (2002). (b) STM topography with atomic resolution of the $(5 \times 1)$ - $\operatorname{Ir}(001)$ surface and (c) top view ball model of this reconstructed surface and characteristic adsorption sites. (b), (c) From Menzel et al., 2012. (d) Symmetry equivalent biatomic zigzag chains on the (5 × 1)-Ir(001) surface as realized by Co. From Dupé et al., 2015. (e) SP-STM topography colorized with the simultaneously obtained differential conductance signal of biatomic $\mathrm{Fe}$ and Co chains grown on $(5 \times 1)-\operatorname{Ir}(001)$; whereas the Fe chain on the left switches its magnetization frequently the magnetic spin spiral ground state of the right Fe chain (see sketch) is fixed by direct exchange coupling to the adjacent ferromagnetic Co chain. Adapted from Menzel et al., 2012. 
preserving the symmetry of the underlying substrate, or zigzag chains can be formed that break the mirror plane within the chain axis [see sketches of the two resulting zigzag chains in Fig. 10(d)]. Such zigzag chains are realized when Co is deposited and they were studied using SP-STM. Similar to the case of the Co chain attached to the step edge of $\mathrm{Pt}(997)$, the Co biatomic chains on $(5 \times 1)-\operatorname{Ir}(001)$ are ferromagnetic with an easy magnetization axis canted with respect to the surface normal (Dupé et al., 2015).

Biatomic Fe chains on the $(5 \times 1)$ - $\operatorname{Ir}(001)$ conserve the symmetry of the substrate, i.e., two orthogonal mirror planes, and the easy axis is restricted to the high-symmetry directions. In contrast to the Co chains, where at $8 \mathrm{~K}$ the magnetic anisotropy energy is sufficiently large to suppress a thermally induced magnetization switching, the magnetic state of the $\mathrm{Fe}$ chain switches on a timescale that is much shorter than the typical SP-STM signal detection time resolution of ms/pixel. Consequently, at zero magnetic field, the spin-polarized contribution to the tunnel current is averaged and vanishes, leading to a homogeneous signal along the chain [see the left chain in Fig. 10(e)], posing a challenge to the experimental investigation of such one-dimensional magnetic chains.

In SP-STM measurements on biatomic Fe chains on $(5 \times 1)$ $\operatorname{Ir}(001)$ at $8 \mathrm{~K}$ a magnetic signal is detected only when the thermal fluctuations of the magnetic state are suppressed either by direct exchange coupling to a stable magnet, as demonstrated in Fig. 10(e) with a ferromagnetic Co chain, or by application of an external magnetic field (Menzel et al., 2012); see Fig. 12(c). The observed magnetic superstructure of about three atomic distances originates from a spin-spiral ground state. As revealed by DFT, this magnetic state results from a competition of direct (nearest-neighbor), indirect (more distant neighbors via substrate), and antisymmetric exchange interactions (Dzyaloshinskii-Moriya). The direct exchange interaction is strongly ferromagnetic for the pairs perpendicular to the chains axis, but only very small along the chains with a similar order of magnitude as exchange between more distant spins and the Dzyaloshinskii-Moriya interaction (Menzel et al., 2012).

Chains by atom manipulation.-The self-organization technique has the advantage of using thermodynamics to achieve a reproducible way of creating a large number of quasi-infinitely long chains. However, it is also totally dependent on the patterning of the substrate. Therefore, the distance between the atoms in the chain is rather fixed by the substrate properties. The mutual exchange interactions between the atoms in the chain cannot be varied easily from the dense, direct-exchange dominated regime into the dilute, indirect itinerant-electron exchange mediated, or so-called RKKY dominated regime. Finally, it cannot be used to create individual spin chains of an absolutely well-defined number of atoms. A different approach is the creation of spin chains by STM-tip induced atom manipulation which is illustrated in Fig. 11.

Using SP-STS (Zhou et al., 2010; Khajetoorians et al., 2012) or IETS (Khajetoorians et al., 2016), the magnetizations or excitations, respectively, of two atoms of the desired chain material in an RKKY-coupled pair can be measured as a function of an external magnetic field. By fitting the data to models using effective spin Hamiltonians (Sec. II.A) it is possible to extract the isotropic $\left(J_{i j}\right)$ and DzyaloshinskiiMoriya $\left(\vec{D}_{i j}\right)$ components of this pairwise RKKY interaction. An example is shown in Figs. 11(a)-11(c) for an Fe atom and an Fe-hydrogen complex on $\operatorname{Pt}(111)$ with increasing separation between them in comparison to DFT. These maps of distance-dependent exchange interactions can then be used in order to tailor and build artificial dilute chains of magnetic atoms on different substrates; see Figs. 11(d)-11(g).

For the example of Fe atoms on $\mathrm{Cu}(111)$ [Fig. 11(d)], it was found that the longitudinal magnetic anisotropy $D$ of the atoms is the dominant energy scale and about an order of magnitude larger than $J_{i j}$ (Khajetoorians et al., 2012), while $\vec{D}_{i j}$ is negligible because of the relatively light substrate and consequently weak spin-orbit interaction. Therefore, this system behaves like an Ising system. By choosing an appropriate interatomic distance, it was possible to stabilize the Néel state in artificial chains with a different number of atoms. A detection by SP-STM was possible either by coupling the first atom to a ferromagnetic island via RKKY interactions (Khajetoorians, Wiebe et al., 2011) [Fig. 11(d)] or, for odd-numbered chains, by stabilizing one state in a weak external magnetic field (Khajetoorians et al., 2012); see Fig. 12(a).

For the heavier substrate $\operatorname{Pt}(111)$ with stronger spin-orbit coupling [Figs. 11(a)-11(c)], the two RKKY contributions $J_{i j}$ and $\vec{D}_{i j}$ are of similar strength and comparable to the strength of the longitudinal magnetic anisotropy $D$ (Khajetoorians et al., 2015). Because of symmetry reasons, the main component $D_{\perp}$ of $\vec{D}_{i j}$ lies in the surface plane and is perpendicular to the displacement vector between the two atoms. Therefore, for weak magnetic field the pair is in a noncollinear spin state and by adjusting the distance $d$, the sign and strength of $D_{\perp}$, and thereby the sense and angle of rotation of the spin from one to the other atom can be tailored. By RKKY coupling the first atoms of an artificial dilute 16-Fe atom chain of appropriate atomic distance to a ferromagnetic Co layer [Fig. 11(e)], it was indeed possible to stabilize a spinspiral state [Fig. $11(\mathrm{~g})]$ as proven by the change in the spinresolved apparent height of the chain atoms when reversing the Co layer; see Fig. 11(f). These results demonstrate that the knowledge of the distance-dependent RKKY interaction in combination with STM-tip induced manipulation allows for a high level of versatility and tunability of spin chains of various elements on various substrates.

Parity effects.-Using self-assembled as well as manipulated antiferromagnetic or noncollinear chains, interesting effects on the number of the atoms in the chain, the so-called parity effects, have been studied. Such parity effects are generally based on the dependence of the strength or orientation of the net magnetic moment of the chain on the parity of the number of the chain atoms.

For example, an antiferromagnetic Ising chain of an odd number of atoms has a nonzero net magnetic moment. In a weak homogeneous magnetic field which is small enough such that the Zeeman energy cannot break the nearestneighbor exchange interactions $\left(B<2\left|J_{\mathrm{NN}}\right| / m\right.$ with the nearest-neighbor interaction $J_{\mathrm{NN}}$ and the modulus of the magnetic moment of each atom $m$ ), the stabilization of 


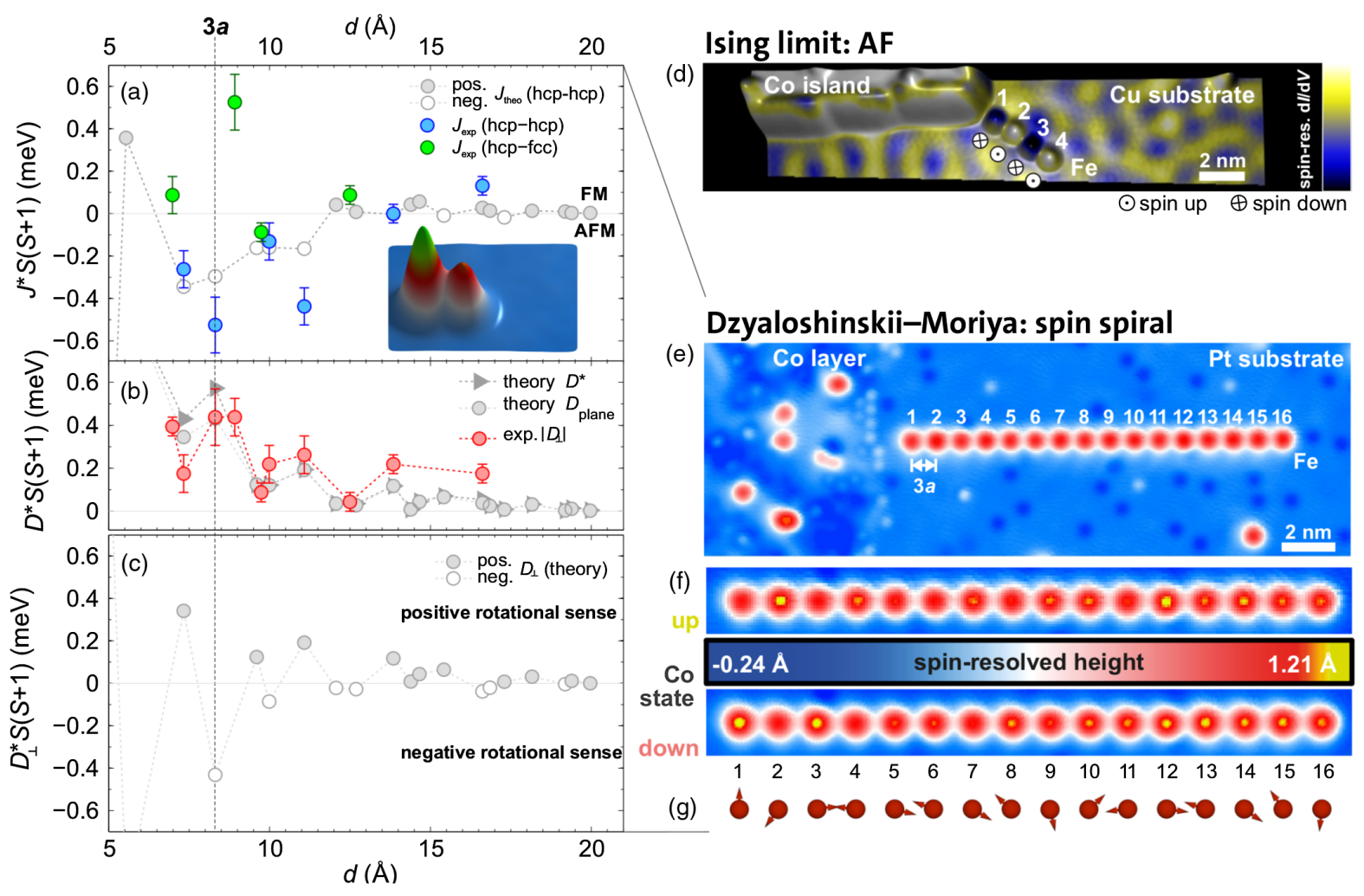

FIG. 11. STM-tip crafted magnetic chains on metallic substrates. (a), (b) Experimentally measured and DFT calculated (a) Heisenberg and (b) Dzyaloshinskii-Moriya components of the RKKY interaction between an Fe atom and an Fe-hydrogen complex on Pt(111) (see inset) as a function of their separation $d$. (c) DFT calculated in-plane component $D_{\perp}$ of the Dzyaloshinskii-Moriya vector which determines the indicated rotational sense of the magnetization in the pair. (a)-(c) Adapted from Khajetoorians et al., 2016. (d) Spinresolved image of a chain of four antiferromagnetically RKKY-coupled Fe atoms on $\mathrm{Cu}(111)$ which are stabilized by the RKKY interaction to a magnetic Co island. Adapted from Khajetoorians, Wiebe et al., 2011. The color reflects the spin orientation of each atom in the chain, as indicated by the symbols. (e), (f) Spin-resolved images of a chain of 16 antiferromagnetically RKKY-coupled Fe atoms on Pt(111) which are stabilized by RKKY interaction to a magnetic Co layer. Adapted from Steinbrecher et al., 2018. (f) The magnetization of the Co layer is magnetized up (top) and down (bottom) resulting in the alignment of the spin of the first chain atom (up and down). (g) Sketch of the approximate spin orientations of the Fe chain atoms.

the Néel state is expected. This state was indeed observed in odd-numbered Fe chains on $\mathrm{Cu}(111)$ using SP-STS in a weak external magnetic field; see Fig. 12(a) (Khajetoorians et al., 2012). In contrast, for the even-numbered Ising chains, the net magnetic moment is zero, such that a homogeneous magnetic field cannot stabilize the Néel state. Depending on whether the system is in the classical or quantum mechanical limit, the resulting state either fluctuates between two degenerate classical states or, respectively, is a quantum superposition. In the former case, the fluctuation is typically much faster than the time resolution of conventional SP-STS, resulting in a loss of the magnetic contrast on chains with an even number of atoms, as indeed observed for Fe chains on $\mathrm{Cu}(111)$; see Fig. 12(a). In this case, the Néel state can still be stabilized by a local RKKY-exchange field acting on the end of the chain, e.g., by RKKY coupling the chain end to a stable ferromagnetic island; see Fig. 11(d). For the quantum mechanical limit, i.e., spin chains largely decoupled from the substrates electron bath (see Sec. IV), even-odd effects have also been studied. For $\mathrm{Mn}$ chains on $\mathrm{Cu}_{2} \mathrm{~N}$ (see Fig. 6) they manifest in the presence or absence of the singlet-triplet excitation for the even- and odd-numbered chains, respectively. For Co chains on $\mathrm{Cu}_{2} \mathrm{~N}$ they are visible as a qualitative difference between the magnetic field-dependent IETS data of even- and oddnumbered chains as shown in Fig. 8(c).

An even-odd effect has also been proposed for short Mn chains that have been manipulated on a ferromagnetic $\mathrm{Ni}(110)$ substrate (Holzberger et al., 2013). Again, there is antiferromagnetic coupling between the chain atoms. However, in this case, the chain atoms are additionally ferromagnetically exchange coupled to the substrate which is magnetized along the chain axis, resulting in a homogeneous exchange field along the chain. Here DFT predicts a Néel state (collinear) oriented along the chain axis for the odd-numbered chains. For the even-numbered chains, the calculations predict a fluctuation between two degenerate noncollinear ground states which are generated by the exchange field from the substrate and the magnetic anisotropy. When the magnetic atoms of a chain on a magnetic substrate are positioned at larger distances the exchange coupling along the chain can be reduced and the magnetic moments of the chain mimic the magnetic structure of the underlying substrate. In the case of Co atoms on a Mn monolayer on W(110) this structure is a spin spiral (Serrate et al., 2010).

When the magnetic state of the chain itself is noncollinear, the even-odd effects generally become more complex. In the 

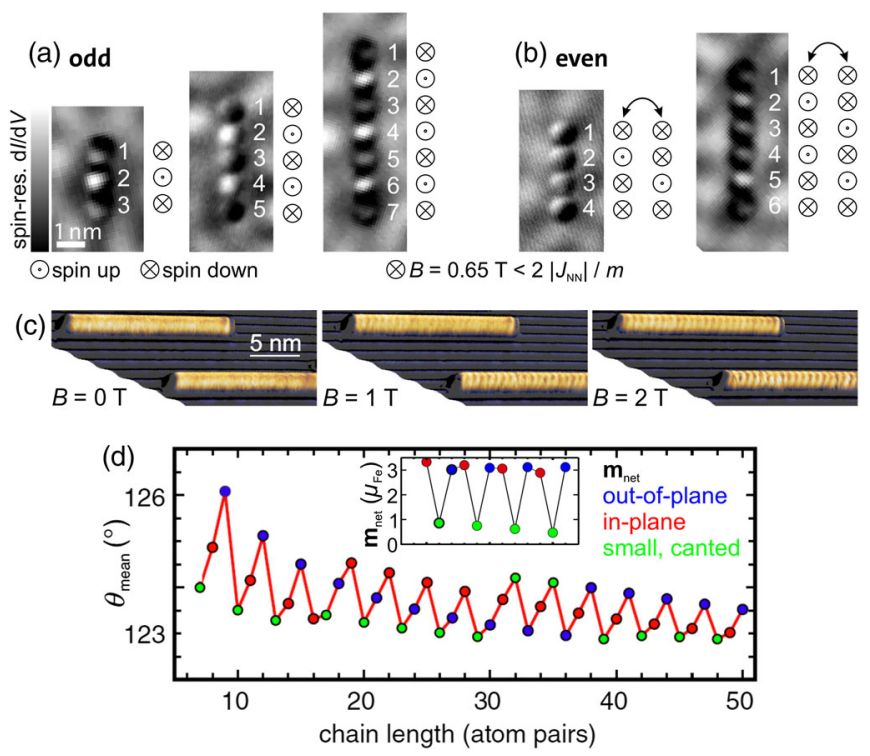

FIG. 12. Parity effects in STM-tip assembled and self-organized spin chains on metallic substrates. (a), (b) Spin-resolved differential conductance images of several (a) odd- and (b) evennumbered artificially constructed $\mathrm{Fe}$ chains on $\mathrm{Cu}(111)$ in the indicated magnetic field. Adapted from Khajetoorians et al., 2012. The resulting Néel states (odd case) and the two states between which the chain fluctuates (even case) are given on the right side of each image. (c) Self-assembled biatomic Fe chains on $(5 \times 1)-\operatorname{Ir}(001)$ as a function of the external out-of-plane magnetic field. (d) Results from micromagnetic simulations showing the chain-length dependent variation of the mean angle and the net magnetic moment. (c), (d) From Menzel et al., 2014.

case of biatomic Fe chains on $(5 \times 1)$ - $\operatorname{Ir}(001)$ the differences between the magnetism of chains with varying lengths manifest in the magnetic field dependence of the amplitude of the spin contrast [see the chains in Fig. 12(c)] (Menzel et al., 2014). Because the period of the spin spiral is nearly three atoms, micromagnetic simulations using the DFT parameters have identified three different types of chains that alternate: they can be classified according to the size and direction of the net magnetic moment of the chain. Depending on the number of atomic pairs of the chain, the net magnetic moment is either large and perfectly out of plane or perfectly in plane, or it is small and has no characteristic direction; see the graph in Fig. 12(d). Even though the magnetic period is given by the competition of the magnetic interactions this parity effect also becomes evident in the mean angle between the Fe atom pairs; see Fig. 12(d). However, the distortions are very small; note that due to the small deviation from $120^{\circ}$ between adjacent magnetic moments there is a long-range pitch in the succession of the three chain types.

\section{B. Spin chains on superconducting substrates}

$1 D$ topological superconductivity.-Recently, chains of magnetic atoms on superconducting substrates were investigated regarding the possibility to achieve the so-called topological superconductivity in one dimension that goes along with the emergence of Majorana bound states (MBS) at the chain's ends. The realization of MBS in solid-state systems is strongly desired because of their peculiar statistical properties. If two MBS are exchanged, they produce a nontrivial phase that is related with the topology of the crossing trajectories. Kitaev (2003) showed that operations with MBS could be used to develop new schemes of quantum computation, reducing operational errors. Additionally, fermion-parity conservation of the topological superconductor removes problems of decoherence that usually limit quantum computations (Kitaev, 2003; Rainis and Loss, 2012). Moreover, the formation of the MBS is a strongly nonlocal effect, as they always come in pairs, one on each end of the chain. Therefore, MBS are expected to be largely immune against local perturbations. These findings have inspired many theoretical works [for a small sample of review topics on this subject see Nayak et al. (2008), Stern (2010), Alicea (2012), Beenakker (2013), Stern and Lindner (2013), Aguado (2017), and Tokura, Kawasaki, and Nagaosa (2017)].

In an instructive toy model, Kitaev showed that MBS will appear at the ends of a one-dimensional and effectively spinless $p$-wave superconductor (Kitaev, 2001) that is adiabatically connected to a 1D topological superconductor (Pientka et al., 2015). Usually, in elemental superconductors, the orbital part of the wave function of the Cooper pairs is described by an $s$ wave that is even under particle interchange and the spin part by the odd singlet state that warrants an odd wave function. In $p$-wave superconductors, the orbital part of the wave function is a $p$ wave that is odd under particle interchange, and the spin part is then even (the Cooper-pair spin is $S=1$ ). The latter enables pairing of effectively spinless electrons, i.e., electrons of the same spin type, such that all low-energy excitations are spinless and the electronic spin can be effectively left out. Unfortunately, the realization of $p$-wave superconductivity is still controversially discussed for the only proposed bulk candidate $\left(\mathrm{Sr}_{2} \mathrm{RuO}_{4}\right)$.

There are two present experimental approaches to realize a 1D topological superconductor. In the first approach, semiconductor nanowires with Rashba-type spin-orbit interaction are coupled to an $s$-wave superconductor and a magnetic field is applied in a direction perpendicular to the spin-orbit effective field (Mourik et al., 2012; Albrecht et al., 2016; Lutchyn et al., 2018). The rationale behind these experiments is to combine the three following ingredients (Pientka et al., 2015): (i) proximity coupling of a 1D electron system to a bulk $s$-wave superconductor in order to transfer superconductivity to the nanowire, circumventing the Mermin-Wagner theorem that implies the impossibility of superconductivity in one dimension (Mermin and Wagner, 1966). (ii) A Zeeman field needed to spin polarize the electron system such that it is essentially spinless. (iii) Strong spin-orbit coupling and/or the impressing of helical spin states in order to enable Cooper pairing of electrons. Different signatures of MBS have been observed in such structures. In April 2018, the Delft group announced that the zero-bias conductance in their wires reached the predicted limit of the quantum of conductance (Zhang et al., 2018), strong evidence for the existence of MBS at the ends of their nanowires.

The second approach is to couple chains of magnetic atoms to $s$-wave superconductors. Here ingredient (ii) can be circumvented by using ferromagnetic spin chains. Also, ingredient (iii) can be achieved either by using materials 
(chain or superconductor) that have an intrinsically large spinorbit interaction (Li et al., 2014) or by inducing noncollinear, e.g., spin-spiral states, in the chain (Choy et al., 2011; Martin and Morpurgo, 2012; Braunecker and Simon, 2013; Klinovaja et al., 2013; Nadj-Perge et al., 2013; Pientka, Glazman, and von Oppen, 2013; Vazifeh and Franz, 2013; Schecter et al., 2016) that also overrides ingredient (ii). The constituents of such spin chains, i.e., the individual magnetic atoms coupled to the surface of the superconductor, already induce states in the energy gap. These states are usually named Yu-ShibaRusinov (YSR) states after their discoverers (Yu, 1965; Shiba, 1968; Rusinov, 1969). In view of their relation and importance for MBS, the properties of the YSR states have been studied in detail. Let us review them briefly before we turn to the description of the few experimental realizations of a spin chain on superconductor systems in the so-called Shiba chain and wire limits (Pientka et al., 2015), where the latter have revealed strong indications for MBS.

YSR states.-YSR states originate from the weakening of the binding of Cooper pairs induced by the magnetic atom on an $s$-wave superconductor. In order to inject or extract a single electron from the superconductor, the electron energy has to be larger than $\Delta$, which can be interpreted as the binding energy of the Cooper pair (de Gennes, 1989; Tinkham, 2004). A magnetic atom in a superconductor can scatter electrons if their energy is larger than $\Delta$, but it can also scatter Cooper pairs. If we assume that $\Delta$ is large enough, spin flips by the magnetic impurity can be safely ignored, and, in first approximation, the effect of the impurity on the conduction electrons can be regarded as that of an exchange field. The exchange field will act on the two electrons of the $s$-wave Cooper pair in a different way, depending on their spin, hence weakening the binding energy of the Cooper pair. As a consequence, it can be easier to break this Cooper pair and the energy to inject or extract an electron will be less than $\Delta$. This can create a state in the energy gap of the superconductor, the YSR state.

After the first experimental verification of YSR states of individual atoms using STS (Yazdani et al., 1997), numerous experimental studies have been performed, revealing effects due to the orbital structure of the atoms (Ji et al., 2008; Ruby et al., 2016; Choi, Rubio-Verdú et al., 2017), the magnetic anisotropy of the atom (Hatter et al., 2015), a reduced dimensionality of some superconductors (Ménard et al., 2015), the competition between Kondo screening and Cooper pairing (Franke, Schulze, and Pascual, 2011; Bauer, Pascual, and Franke, 2013), and the spin polarization of the YSR state (Cornils et al., 2017). Because of the orbital structure of the magnetic atom, there are spatial variations of the exchange field produced by the atom, which induce a marked shape of the spatial distribution of the YSR state. This was recently revealed in STM measurements of $\mathrm{Cr}$ and $\mathrm{Mn}$ impurities on Pb surfaces (Ruby et al., 2016; Choi, RubioVerdú et al., 2017). Figure 13(a) shows the multiple YSR states originating from the hybridization of the orbitals of the $\mathrm{Cr}$ atom with those of the surrounding $\mathrm{Pb}$ atoms.

Using SP-STS, the theoretically predicted spin polarization of the YSR states of individual magnetic atoms on a superconductor has been experimentally detected (Cornils et al., 2017). To this end single $\mathrm{Fe}$ atoms on the $(3 \times 3)$ oxygen (a)

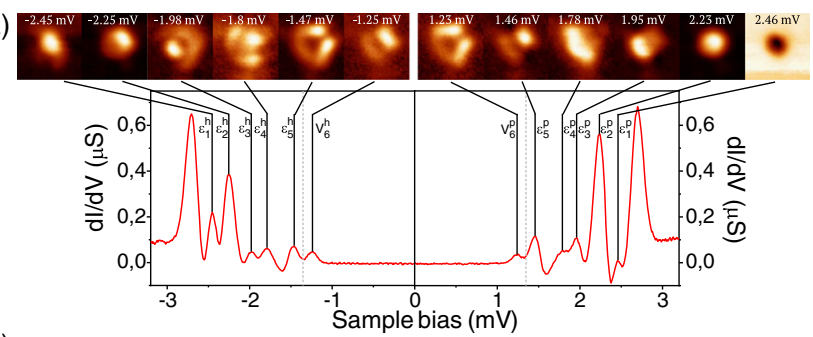

(b)
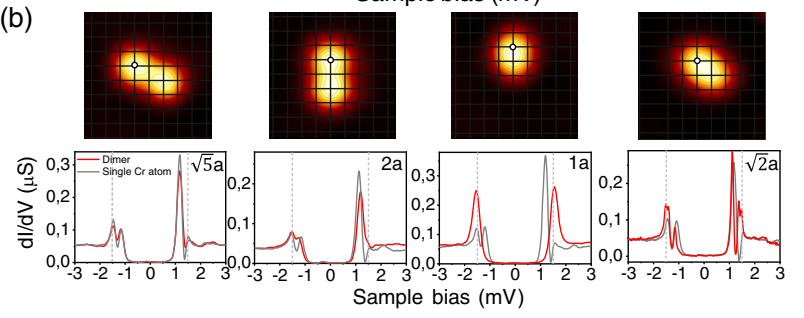

FIG. 13. YSR states in single $\mathrm{Cr}$ atoms on $\mathrm{Pb}(111)$ and $\mathrm{Cr}$ dimers on a $\beta$ - $\mathrm{Bi}_{2} \mathrm{Pd}$ superconductor. (a) Multiple YSR states are shown over a single $\mathrm{Cr}$ atom on $\mathrm{Pb}(111)$, originating from a hybridized orbital structure of $\mathrm{Cr}$ with nearest-neighbor $\mathrm{Pb}$ atoms. Differential conductance maps at the peak positions of the YSR states show the hybridized orbital features. From Choi, RubioVerdú et al., 2017. (b) Dependence of YSR states on the spacing of $\mathrm{Cr}$ atoms in dimers on $\beta$ - $\mathrm{Bi}_{2} \mathrm{Pd}$. Separations from left to right: $\sqrt{5}, 2,1$, and $\sqrt{2}$ by unit cell distances. All dimers were formed by STM-tip induced atomic manipulation. The top panels show the topographic images of the dimers, and the bottom panels show the corresponding differential conductances measured on one of the two atoms in the dimer (red lines) and on a single atom for comparison (gray lines). Adapted from Choi et al., 2018.

reconstruction on $\mathrm{Ta}(100)$ have been magnetized in a small external magnetic field which was weaker than the critical field of the superconducting substrate. The SP-STS data, taken with a magnetic tip that was thoroughly characterized regarding its spin polarization on a reference system, showed the expected sign change in the spin polarization between the electron and the hole parts of the YSR state (Cornils et al., 2017).

Shiba chain limit.-When dilute arrays, e.g., chains, of transition metal magnetic atoms are assembled on a superconductor, the YSR states start to overlap and can eventually form so-called Shiba bands. Here it is assumed that the atoms are sufficiently separated, such that direct hopping between the atom's $d$ levels can be neglected, which is the so-called Shiba chain limit. If the Shiba band is close to the Fermi level and broad enough to overlap with it, topological superconductivity can evolve (Pientka et al., 2015). Some recent works, therefore, probe and characterize the interactions between YSR states with respect to the formation of such a Shiba band (Choi et al., 2018; Kezilebieke et al., 2018; Ruby et al., 2018). The investigation of pairs of $\mathrm{Mn}$ atoms on $\mathrm{Pb}$ (001) reveals the formation of symmetric and antisymmetric combinations of YSR states which is studied as a function of the orientation of the pairs with respect to the orbital shape of the individual YSR states (Ruby et al., 2018). Kezilebieke et al. (2018) investigated the interaction of the YSR states of magnetic molecules on a $\mathrm{NbSe}_{2}$ substrate. Here the formation of coupled YSR states is visible for relatively distant molecules. This is facilitated by the large spatial extent of the YSR 
states due to the 2D character of superconductivity in this material (Frindt, 1972; Gerbold et al., 2015; Xi et al., 2015; Ugeda et al., 2016).

Choi et al. (2018) studied pairs of $\mathrm{Cr}$ atoms on $\beta-\mathrm{Bi}_{2} \mathrm{Pd}$, a type-II superconductor with critical temperature of $5.4 \mathrm{~K}$ (Imai et al., 2012) that has been shown to contain topological surface states (Sakano et al., 2015) characterized by a unique superconducting gap despite its multiband structure (Herrera et al., 2015); see Fig. 13(b). A single $\mathrm{Cr}$ atom on $\beta-\mathrm{Bi}_{2} \mathrm{Pd}$ produces YSR states (Choi et al., 2018). When a second $\mathrm{Cr}$ atom was approached along the [100] direction of the surface, the YSR states shifted and broadened. As the distance was shortened, there was a clear shift to higher energy, driving the YSR state toward the quasiparticle continuum edge. At very short distances, leading to the formation of a $\mathrm{Cr}_{2}$ dimer, the YSR peak disappeared into the continuum. These data were interpreted as the disappearance of the localized magnetic moment over the $\mathrm{Cr}-\mathrm{Cr}$ system, which implies an antiferromagnetic interaction between the two magnetic moments. For pairs oriented along the [1-10] direction, the YSR states split, indicating a hybridization of the YSR states consistent with a ferromagnetic coupling of the $\mathrm{Cr}$ magnetic moments (Flatté, 2000; Choi et al., 2018).

Along these lines, artificial chains of magnetic atoms with YSR states were assembled on a superconducting substrate using STM-tip induced atom manipulation. Kamlapure et al. (2018) investigated the coupling of the YSR states in artificial chains of $\mathrm{Fe}$ atoms assembled on the $(3 \times 3)$ oxygen reconstruction of $\mathrm{Ta}(100)$; see Fig. 14. While pairs of $\mathrm{Fe}$ adatoms showed a negligible interaction of the YSR states due to a relatively large $\mathrm{Fe}-\mathrm{Fe}$ distance, the interaction was increased by the manipulation of subsurface interstitial $\mathrm{Fe}$ atoms in the center between the two Fe adatoms, as proven by the shift of the YSR states. Motivated by this effect, chains of $\mathrm{Fe}$ adatoms and subsurface interstitial $\mathrm{Fe}$ atoms were assembled [Figs. 14(a) and 14(b)] and investigated concerning the formation of a YSR band; see Figs. 14(c) and 14(d)]. Even though there is a considerable interaction between the YSR states as visible from the change in the YSR state energy when one of the chain atoms is switched into a nonmagnetic state [Figs. 14(b)-14(d)], the YSR state energies are distributed inhomogeneously along the chain [Fig. 14(d)] indicating considerable electronic disorder, which prevents the formation of a YSR band. So far, an experimental realization of a clean YSR band in a chain of dilute magnetic atoms on a superconductor, i.e., within the Shiba band limit, is still missing.

Wire limit.-When the transition metal atoms in a chain are more densely packed, direct hopping between the $d$ levels of the atoms can no longer be neglected. If the resulting $d$ band crosses the Fermi level, it has to be considered in the description of the low-energy phenomena. This limit is called the wire limit (Pientka et al., 2015). Two systems reported in the literature so far show evidence for MBS at the ends of spin chains in this limit. Nadj-Perge et al. (2014) investigated Fe chains attached to clusters on the $\mathrm{Pb}(110)$ surface. These spin chains are formed after room-temperature deposition of $\mathrm{Fe}$, followed by annealing. With the help of DFT, the most probable structure of the chain was found to be a three-layer Fe zigzag chain partially submerged in the $\mathrm{Pb}$. Spin-resolved STS revealed a contrast consistent with a ferromagnetic
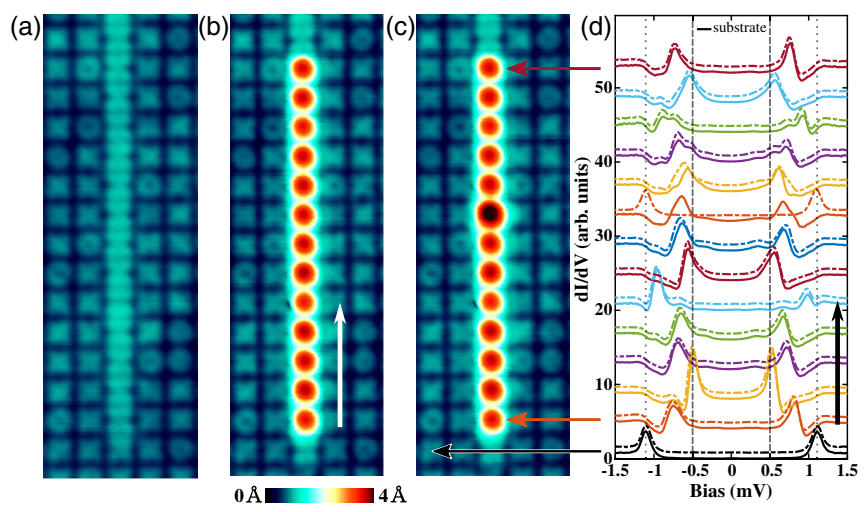

FIG. 14. STM-tip assembled chain of weakly coupled Fe atoms on $\mathrm{Ta}(100)-(3 \times 3) \mathrm{O}$. (a) Assembled chain of subsurface interstitial Fe atoms. (b) Thirteen Fe atoms have been assembled on the surface along the subsurface atoms, such that each Fe atom is linked by a subsurface Fe atom. (c) The sixth atom from the top has been switched into a nonmagnetic state. (d) Comparison of differential conductance spectra taken with a superconducting tip on every Fe adatom and on the substrate, with all atoms in the magnetic state (solid lines) and the sixth atom in the nonmagnetic state (dashed lines). The dashed (dotted) vertical lines indicate the sample Fermi level (sample coherence peak). Adapted from Kamlapure et al., 2018.

alignment of the topmost $\mathrm{Fe}$ atoms in the chain, and it was concluded with the help of DFT that the chain is in a ferromagnetic state. STS revealed a zero-bias peak within a length of 1 to $2 \mathrm{~nm}$ from the end of the chains which was interpreted as the signature of a MBS. The zero-bias peak in this system was reproduced by two other groups (Ruby et al., 2015; Pawlak et al., 2016). Ruby et al. pointed out that it is present only for some of the chains, and moreover, as revealed by higher resolution studies, that the zero-bias peak overlaps with a low-energy resonance that was tentatively attributed to the coherence peak of the induced topological gap (Ruby et al., 2015). A study performed at even lower temperatures (Feldman et al., 2017) on the same system showed that the zero-bias peak has no detectable splitting, is particle-hole symmetric for some of the chains and asymmetric for others, and has a significant spectral weight in the substrate. Finally, spin-resolved STS of the zero-bias peak (Jeon et al., 2017) reveals signals that are symmetric with respect to bias reversal, in contrast to the antisymmetric signals observed for YSR bands. The absence of splitting and particle-hole symmetry strongly support the MBS interpretation of the zero-bias peak.

Experiments of $\mathrm{Co}$ chains on $\mathrm{Pb}$ (110) with a similar morphology and signature of ferromagnetic order as for the case of Fe chains yielded a zero-bias signal delocalized along the chain. The lack of localization at the edges precludes the presence of MBS (Ruby et al., 2017). Tight-binding calculations for this system indicated an even number of Fermi level crossings of the spin-orbit-split bands, in contrast to an odd number found for the Fe chain system (Ruby et al., 2017), which would explain the absence and presence of a topological phase for the $\mathrm{Co}$ and $\mathrm{Fe}$ systems, respectively.

The experimental difficulty in finding a consistent signature of MBS for some of the chains of the $\mathrm{Fe} / \mathrm{Pb}(110)$ system which have been investigated (Ruby et al., 2015; Feldman 
et al., 2017) most probably originates in an imperfect atomic structure as evident from the variance in topographic features found at the ends of the chains. This problem can be circumvented by the investigation of artificial chains that are built by STM-tip induced manipulation. Unfortunately, for the system $\mathrm{Pb}(110)$, this procedure turned out impossible. However, Kim et al. (2018) were able to manipulate virtually atomically perfect chains of several tens of Fe atoms on the (0001) surface of the strong-spin-orbit coupling superconductor Re. Spin-resolved STS of chains of different numbers of Fe atoms positioned on the close-packed hcp hollow sites of the $\operatorname{Re}(0001)$ surface reveals a noncollinear magnetic state. Recent calculations corroborate the possibility of obtaining spin spirals in this system as well as emphasizing the need for describing high-order spin interactions (Lászlóffy et al., 2019). STS furthermore shows zero-bias conductivity localized in a region of half a nm width at both chain ends, which disappears for chains with less than $9 \mathrm{Fe}$ atoms. With the help of tight-binding model calculations based on parameters obtained from $a b$ initio calculations, which predict that the chains are in the topologically superconducting state, the zerobias conductivity was interpreted as an indication for MBS at the chain ends. Manipulation of a single-atom defect to the end of a short Fe chain interestingly revealed that zero-bias peaks can be generated by such defects, stressing that a full control of the chain composition is essential in order to rule out trivial effects inducing zero-bias peaks that can potentially be mistaken for MBS.

\section{OUTLOOK}

Spin chains are the paradigm of quantum phenomena. Entanglement, correlation, and decoherence are properties inherent to spin chains. In this Colloquium, we showed an overview of these phenomena in different contexts. We specifically focused on scanning probes that, besides atomic manipulation, permit us to obtain detailed spectroscopic information on individual chains of precise length and composition.

Complementary to other techniques such as atom traps or molecular crystals, the STM manipulation of atoms on surfaces offers an extremely controlled way of creating structures with tailored properties. The substrate is the main constraint in these systems, which, on the one hand, gives to environmental perturbations on the properties of the spin chain. On the other hand, the use of substrates makes it possible to eventually encapsulate and create devices, giving us ideas on how to create a useful technology out of the quantum properties of entangled spins.

The approaches presented in this Colloquium are related to many exciting research areas such as quantum information science. Let us briefly mention some of the interesting connections.

Spintronics.-The studies we presented here bear direct relation to the possibility of using spin instead of charge in solid-state devices. Atom manipulation grants new capabilities of creating devices with atomic precision. The rich spectra of spin chains and the different ways to access them via electronic currents that we have presented in this Colloquium show that indeed operation can be performed at the atomic level in spins conveniently coupled to other spins and decoupled from the degrees of freedom of the substrate. Spin chains have been used to realize all spin-based logic operations (Khajetoorians, Wiebe et al., 2011), to serve as tiny storage units of memory when conveniently arranged using antiferromagnetic couplings (Loth et al., 2012), and inelastic effects have been shown to be an effective way of inducing spin torque (Delgado, Palacios, and Fernández-Rossier, 2010; Loth, von Bergmann et al., 2010; Khajetoorians et al., 2013). We can easily envisage new applications of spin chains in spintronic devices by using resonant excitation of spin in timedependent approaches (Baumann, Paul et al., 2015), or combining them with the rich world of semiconductors (see Sec. IV.C) to produce new devices. Indeed, a new type of spinbased transistor was suggested (Marchukov et al., 2016). They showed that by switching on and off the entanglement with parts of the spin chain using local spin excitations, a spinbased transistor can be achieved.

Quantum communication.-Using quantum mechanics to encode information and process it is a tantalizing field with enormous possibilities. Recent suggestions show that information can be indeed transmitted with high fidelity in spin chains. Khaneja and Glaser (2002) used an Ising spin chain to serve as a transmitting line of radio-frequency pulses that drive single-spin information. Bose (2003) used a Heisenberg spin chain by putting to work its excitation spectrum. There it was shown that an excitation in one extreme of the chain is partially transmitted to the other edge of the chain. These results are regardless of the sign of the Heisenberg coupling because they are a consequence of the full spectrum of excitations. Perfect transmission has proved to take place in customized spin chains. Christandl et al. (2004) proved that perfect fidelity over long distance is obtained when using an $X Y$-coupled spin chain and also a Heisenberg spin chain where the couplings are modulated by an external magnetic field. Karbach and Stolze (2005) showed that by tuning the parameters of the spin chain one can actually obtain perfect transmission. Contrary to intuition, there is a full class of inhomogeneously coupled chains that even allows for small variations along the chain, permitting the transmission of information with perfect fidelity. Karbach and Stolze (2005) further showed that transmission over considerable distances can be achieved at arbitrary temperatures for genuinely entangled states, giving rise to many technological options. Quantum fluctuations can be put to work in transmitting information in an effective way (Banchi et al., 2017). Indeed, a new proposal uses a small external magnetic field to tune a given quantum phase of the connecting spin chain and in this way control the transmission of information (Banchi et al., 2017).

Quantum computing.-Qubits and operations on qubits need to be performed within the quantum coherence time. Using a solid device is probably a difficult strategy due to the large number of degrees of freedom and interactions that will necessary perturb the acting spins. Decoupling the spins is a strategy that seems to be working to have access to these quantities with the STM, thus opening the door to applications of solid-supported spin chains in quantum computing. We have seen in the cases studied so far that the solid and more generally, the environment, becomes part of the quantum 
system. Typically this is not beneficial because it can lead to faster decoherences and other effects that destroy the superposition states needed for quantum computation. However, the substrate can be beneficial. This is clearly seen in the case of a superconducting substrate, where it is the substrate that develops extraordinary topological properties leading to Majorana edge states that can be potentially used for topological quantum computing. Furthermore, superconductors have gaps that partially decouple the spin chain, reducing excitation and other undesirable phenomena. Choosing the substrate is an important aspect of the future developments of spin chains. (Mermin, 2007; Bruss and Leuchs, 2019)

Quantum simulations.-This is a fascinating field where atom traps are making much progress as we briefly mentioned in the Introduction. Spins on surfaces can also be used by experimentally revealing the solutions to model Hamiltonians that represent the behavior of matter on the very-low-temperature scale. Indeed, the rich world of spin-based Hamiltonians that have seen the light since the introduction of the Bethe ansatz and integrable models based on spin chains gives us new methods to undertake the exploration of quantum matter. The study of these systems with the new tools offered by the STM is an intriguing field for future research (Georgescu, Ashhab, and Nori, 2014; Gross and Bloch, 2017)

Quantum sensors.-The limits of metrology have been further extended by the use of quantum measurements. Smaller quantities are accessible using quantum effects thanks to the interference aspects of superposition states. A recent experiment using electron-spin resonance with an STM shows that atomic spins can be used as extremely precise and sensitive sensors (Natterer et al., 2017). The experiment uses the shift in the electronic current resonance peak when the STM tip is on top of an Fe atom as a detector of the magnetic dipolar interaction with a nearby Ho atom. By fitting the known $1 / r$ law of the dipolar interaction at a different Fe-Ho distance, they are able to measure the intrinsic magnetic moment of a single Ho atom. This experiment shows that using quantum effects, the energy scale is in the sub- $\mu$-eV range (T. Choi et al., 2017). From measuring minute magnetic fields to having access to the very-low-energy scale of superconducting gaps, quantum metrology can have a strong impact on biosensors, industry, and creating new standards of measurements, as is already the case with the redefinition of the SI unit system in 2018.

In summary, spin chains on solid surfaces have become accessible and are interesting objects of research. This new research field is rich and lively due to the extraordinary prospects of the scanning tunneling microscope. New developments are further advancing the field. These developments include the possibility to measure forces and currents concurrently, permitting the analysis of magnetic structures with unprecedented accuracy. Furthermore, the newly timeresolved technics are expanding our insight into the basic understanding and ability to manipulate spins on the atomic scale. These advances have enabled researchers to measure lifetimes and coherence times of spins on surfaces with unprecedented accuracy. We are gathering new insight into the dynamics of superposition states and interactions at play. We can now explore new phases of matter, particularly the newly discovered topological phases. Spin-chain research is a fundamental rich field for future exploration in all of these topics.

\section{ACKNOWLEDGMENTS}

D.-J.C. and N.L. acknowledge financial support from MICINN (RTI2018-097895-B-C44) and FEDER funds. J.W. and K. v. B. received funding from the Deutsche Forschungsgemeinschaft (DFG, German Research foundation)-SFB668. A.F. O. acknowledges support from the Netherlands Organisation for Scientific Research (NWO) and the European Research Council (ERC Starting Grant No. 676895 "SPINCAD"). A. J.H acknowledges support from the Institute for Basic Science under IBS-R027-D1.

\section{REFERENCES}

Affleck, I., 1989, J. Phys. Condens. Matter 1, 3047.

Affleck, I., T. Kennedy, E. H. Lieb, and H. Tasaki, 1987, Phys. Rev. Lett. 59, 799.

Aguado, R., 2017, Riv. Nuovo Cimento Soc. Ital. Fis. 40, 523.

Albrecht, S. M., A. P. Higginbotham, M. Madsen, F. Kuemmeth, T. S. Jespersen, J. Nygaard, P. Krogstrup, and C. M. Marcus, 2016, Nature (London) 531, 206.

Alicea, J., 2012, Rep. Prog. Phys. 75, 076501.

Auerbach, A., 1994, Interacting Electrons and Quantum Magnetism (Springer-Verlag, Berlin).

Balashov, T., et al., 2009, Phys. Rev. Lett. 102, 257203.

Banchi, L., J. Fernández-Rossier, C. F. Hirjibehedin, and S. Bose, 2017, Phys. Rev. Lett. 118, 147203.

Bartels, L., G. Meyer, and K.-H. Rieder, 1997, Phys. Rev. Lett. 79, 697.

Bauer, J., J. I. Pascual, and K. J. Franke, 2013, Phys. Rev. B 87, 075125.

Baumann, S., W. Paul, T. Choi, C. P. Lutz, A. Ardavan, and A. J. Heinrich, 2015, Science 350, 417.

Baumann, S., et al., 2015, Phys. Rev. Lett. 115, 237202.

Beenakker, C., 2013, Annu. Rev. Condens. Matter Phys. 4, 113.

Bethe, H. A., 1931, Z. Phys. 71, 205.

Blügel, S., and G. Bihlmayer, 2007, Magnetism of low-dimensional systems: Theory (John Wiley \& Sons, New York).

Bode, M., 2003, Rep. Prog. Phys. 66, 523.

Bose, S., 2003, Phys. Rev. Lett. 91, 207901.

Bougourzi, A. H., M. Karbach, and G. Müller, 1998, Phys. Rev. B 57, 11429.

Braunecker, B., and P. Simon, 2013, Phys. Rev. Lett. 111, 147202.

Bruss, D., and G. Leuchs, 2019, Quantum Information (Wiley-VCH, Weinheim, Germany)

Bryant, B., A. Spinelli, J. Wagenaar, M. Gerrits, and A. Otte, 2013, Phys. Rev. Lett. 111, 127203.

Bryant, B., R. Toskovic, A. Ferran, J. L. Lado, A. Spinelli, J. Fernández-Rossier, and A. F. Otte, 2015, Nano Lett. 15, 6542.

Caneschi, A., D. Gatteschi, N. Lalioti, C. Sangregorio, R. Sessoli, G. Venturi, A. Vindigni, A. Rettori, M. G. Pini, and M. A. Novak, 2001, Angew. Chem., Int. Ed. Engl. 40, 1760.

Caux, J.-S., and R. Hagemans, 2006, J. Stat. Mech. 12, P12013.

Chen, X., Z.-C. Gu, Z.-X. Liu, and X.-G. Wen, 2012, Science 338, 1604.

Chilian, B., A. A. Khajetoorians, S. Lounis, A. T. Costa, D. L. Mills, J. Wiebe, and R. Wiesendanger, 2011, Phys. Rev. B 84, 212401 
Choi, D.-J., C. G. Fernández, E. Herrera, C. Rubio-Verdú, M. M. Ugeda, I. Guillamón, H. Suderow, J. I. Pascual, and N. Lorente, 2018, Phys. Rev. Lett. 120, 167001.

Choi, D.-J., and N. Lorente, 2018, in Handbook of Materials Modeling: Applications: Current and Emerging Materials, edited by W. Andreoni and S. Yip (Springer International Publishing, Cham), pp. 1-32.

Choi, D.-J., R. Robles, J.-P. Gauyacq, C. Rubio-Verdú, N. Lorente, and J. I. Pascual, 2016, J. Phys. Condens. Matter 28, 23 LT01.

Choi, D.-J., R. Robles, J.-P. Gauyacq, M. Ternes, S. Loth, and N. Lorente, 2016, Phys. Rev. B 94, 085406.

Choi, D.-J., R. Robles, S. Yan, J. A. J. Burgess, S. Rolf-Pissarczyk, J.-P. Gauyacq, N. Lorente, M. Ternes, and S. Loth, 2017, Nano Lett. 17, 6203.

Choi, D.-J., C. Rubio-Verdú, J. de Bruijckere, M. M. Ugeda, N. Lorente, and J. I. Pascual, 2017, Nat. Commun. 8, 15175.

Choi, T., W. Paul, S. Rolf-Pissarczyk, A. J. Macdonald, F. D. Natterer, K. Yang, P. Willke, C.P. Lutz, and A. J. Heinrich, 2017, Nat. Nanotechnol. 12, 420.

Choy, T.-P., J. M. Edge, A. R. Akhmerov, and C. W. J. Beenakker, 2011, Phys. Rev. B 84, 195442.

Christandl, M., N. Datta, A. Ekert, and A. J. Landahl, 2004, Phys. Rev. Lett. 92, 187902.

Cohen-Tannoudji, C., J. Dupont-Roc, and G. Grynberg, 1998, AtomPhoton Interactions: Basic Processes and applications (Wiley and Sons, Inc., New York).

Cornils, L., A. Kamlapure, L. Zhou, S. Pradhan, A. A. Khajetoorians, J. Fransson, J. Wiebe, and R. Wiesendanger, 2017, Phys. Rev. Lett. 119, 197002.

Crain, J. N., and D. T. Pierce, 2005, Science 307, 703.

de Gennes, P.-G., 1989, Superconductivity of Metals and Alloys (Addison-Wesley, Reading, MA).

Delgado, F., and J. Fernández-Rossier, 2017, Prog. Surf. Sci. 92, 40. Delgado, F., J. J. Palacios, and J. Fernández-Rossier, 2010, Phys. Rev. Lett. 104, 026601.

des Cloizeaux, J., and J. J. Pearson, 1962, Phys. Rev. 128, 2131.

Deuretzbacher, F., D. Becker, J. Bjerlin, S. M. Reimann, and L. Santos, 2014, Phys. Rev. A 90, 013611.

Dmitriev, D. V., V. Y. Krivnov, and A. A. Ovchinnikov, 2002, Phys. Rev. B 65, 172409.

Do, E. H., and H. W. Yeom, 2015, Phys. Rev. Lett. 115, 266803.

Dupé, B., J. E. Bickel, Y. Mokrousov, F. Otte, K. von Bergmann, A. Kubetzka, S. Heinze, and R. Wiesendanger, 2015, New J. Phys. 17, 023014.

Dzyaloshinsky, I., 1958, J. Phys. Chem. Solids 4, 241.

Eigler, D. M., and E. K. Schweizer, 1990, Nature (London) 344, 524. Erwin, S. C., and F. J. Himpsel, 2010, Nat. Commun. 1, 58.

Faddeev, L. D., 1996, "How algebraic Bethe ansatz works for integrable model," arXiv:hep-th/9605187.

Feldman, B. E., M. T. Randeria, J. Li, S. Jeon, Y. Xie, Z. Wang, I. K. Drozdov, B. Andrei Bernevig, and A. Yazdani, 2017, Nat. Phys. 13, 286.

Ferrón, A., F. Delgado, and J. Fernández-Rossier, 2015, New J. Phys. 17, 033020.

Fert, A., and P. M. Levy, 1980, Phys. Rev. Lett. 44, 1538.

Flatté, M. E., 2000, Phys. Rev. B 61, R14920.

Flippen, R. B., and S. A. Friedberg, 1963, J. Chem. Phys. 38, 2652.

Fölsch, S., J. Martínez-Blanco, J. Yang, K. Kanisawa, and S. C. Erwin, 2014, Nat. Nanotechnol. 9, 505.

Fölsch, S., J. Yang, C. Nacci, and K. Kanisawa, 2009, Phys. Rev. Lett. 103, 096104.

Franke, K. J., G. Schulze, and J. I. Pascual, 2011, Science 332, 940.

Frindt, R. F., 1972, Phys. Rev. Lett. 28, 299.
Gambardella, P., M. Blanc, H. Brune, K. Kuhnke, and K. Kern, 2000, Phys. Rev. B 61, 2254.

Gambardella, P., A. Dallmeyer, K. Maiti, M. C. Malagoli, W. Eberhardt, K. Kern, and C. Carbone, 2002, Nature (London) 416, 301 .

Gauyacq, J. P., and N. Lorente, 2011, Phys. Rev. B 83, 035418.

Gauyacq, J.-P., and N. Lorente, 2015, J. Phys. Condens. Matter 27, 455301.

Gauyacq, J.-P., S. M. Yaro, X. Cartoixà, and N. Lorente, 2013, Phys. Rev. Lett. 110, 087201.

Gawronski, H., M. Mehlhorn, and K. Morgenstern, 2008, Science 319, 930.

Georgescu, I. M., S. Ashhab, and F. Nori, 2014, Rev. Mod. Phys. 86, 153.

Gohlke, D., R. Mishra, O. D. Restrepo, D. Lee, W. Windl, and J. Gupta, 2013, Nano Lett. 13, 2418.

Gross, C., and I. Bloch, 2017, Science 357, 995.

Gu, Z.-C., and X.-G. Wen, 2009, Phys. Rev. B 80, 155131.

Haldane, F. D. M., 1983, Phys. Rev. Lett. 50, 1153.

Haldane, F. D. M., 2017, Rev. Mod. Phys. 89, 040502.

Haseda, T., and A. Miedema, 1961, Physica (Utrecht) 27, 1102.

Hashimoto, K., C. Sohrmann, J. Wiebe, T. Inaoka, F. Meier, Y. Hirayama, R. A. Römer, R. Wiesendanger, and M. Morgenstern, 2008, Phys. Rev. Lett. 101, 256802.

Hatter, N., B. W. Heinrich, M. Ruby, J. I. Pascual, and K. J. Franke, 2015, Nat. Commun. 6, 8988.

Heinrich, A. J., J. A. Gupta, C. P. Lutz, and D. M. Eigler, 2004, Science 306, 466.

Heinz, K., and L. Hammer, 2009, Prog. Surf. Sci. 84, 2.

Heinze, S., K. von Bergmann, M. Menzel, J. Brede, A. Kubetzka, R. Wiesendanger, G. Bihlmayer, and S. Blügel, 2011, Nat. Phys. 7, 713.

Hermenau, J., S. Brinker, M. Marciani, M. Steinbrecher, M. dos Santos Dias, R. Wiesendanger, S. Lounis, and J. Wiebe, 2019, Nat. Commun. 10, 2565.

Herrera, E., et al., 2015, Phys. Rev. B 92, 054507.

Hewson, A., 1993, The Kondo problem to heavy fermions (Cambridge University Press, Cambridge, England).

Himpsel, F. J., J. E. Ortega, G. J. Mankey, and R. F. Willis, 1998, Adv. Phys. 47, 511.

Hirjibehedin, C. F., C.-Y. Lin, A. F. Otte, M. Ternes, C. P. Lutz, B. A. Jones, and A. J. Heinrich, 2007, Science 317, 1199.

Hirjibehedin, C. F., C. P. Lutz, and A. J. Heinrich, 2006, Science 312, 1021.

Ho, W., 2002, J. Chem. Phys. 117, 11033.

Hoffmann, M., and S. Blügel, 2018, arXiv:1803.01315.

Holzberger, S., T. Schuh, S. Blügel, S. Lounis, and W. Wulfhekel, 2013, Phys. Rev. Lett. 110, 157206.

Horodecki, R., P. Horodecki, M. Horodecki, and K. Horodecki, 2009, Rev. Mod. Phys. 81, 865.

Hulthén, L., 1938, Ark. Mat. Ast. Fys. (Sweden) 26A, 1.

Imai, Y., F. Nabeshima, T. Yoshinaka, K. Miyatani, R. Kondo, S. Komiya, I. Tsukada, and A. Maeda, 2012, J. Phys. Soc. Jpn. 81, 113708.

Jeon, S., Y. Xie, J. Li, Z. Wang, B. A. Bernevig, and A. Yazdani, 2017, Science 358, 772.

Ji, S.-H., et al., 2008, Phys. Rev. Lett. 100, 226801.

Kamlapure, A., L. Cornils, J. Wiebe, and R. Wiesendanger, 2018, Nat. Commun. 9, 3253.

Karbach, M., K. Hu, and G. Müller, 1998, Comput. Phys. 12, 565.

Karbach, M., G. Müller, A.H. Bougourzi, A. Fledderjohann, and K.-H. Mütter, 1997, Phys. Rev. B 55, 12510.

Karbach, P., and J. Stolze, 2005, Phys. Rev. A 72, 030301. 
Kasuya, T., 1956, Prog. Theor. Phys. 16, 45.

Kezilebieke, S., M. Dvorak, T. Ojanen, and P. Liljeroth, 2018, Nano Lett. 18, 2311.

Khajetoorians, A. A., B. Chilian, J. Wiebe, S. Schuwalow, F. Lechermann, and R. Wiesendanger, 2010, Nature (London) 467, 1084.

Khajetoorians, A. A., S. Lounis, B. Chilian, A. T. Costa, L. Zhou, D. L. Mills, J. Wiebe, and R. Wiesendanger, 2011, Phys. Rev. Lett. 106, 037205.

Khajetoorians, A. A., T. Schlenk, B. Schweflinghaus, M. dos Santos Dias, M. Steinbrecher, M. Bouhassoune, S. Lounis, J. Wiebe, and R. Wiesendanger, 2013, Phys. Rev. Lett. 111, 157204.

Khajetoorians, A. A., M. Steinbrecher, M. Ternes, M. Bouhassoune, M. dos Santos Dias, S. Lounis, J. Wiebe, and R. Wiesendanger, 2016, Nat. Commun. 7, 10620.

Khajetoorians, A. A., M. Valentyuk, M. Steinbrecher, T. Schlenk, A. Shick, J. Kolorenc, A. I. Lichtenstein, T. O. Wehling, R. Wiesendanger, and J. Wiebe, 2015, Nat. Nanotechnol. 10, 958.

Khajetoorians, A. A., J. Wiebe, B. Chilian, S. Lounis, S. Blügel, and R. Wiesendanger, 2012, Nat. Phys. 8, 497.

Khajetoorians, A. A., J. Wiebe, B. Chilian, and R. Wiesendanger, 2011, Science 332, 1062.

Khajetoorians, A. A., et al., 2013, Science 339, 55.

Khaneja, N., and S. J. Glaser, 2002, Phys. Rev. A 66, 060301.

Kim, H., A. Palacio-Morales, T. Posske, L. Rózsa, K. Palotás, L. Szunyogh, M. Thorwart, and R. Wiesendanger, 2018, Sci. Adv. 4, eaar5251.

Kitaev, A., 2003, Ann. Phys. (Amsterdam) 303, 2.

Kitaev, A. Y., 2001, Phys. Usp. 44, 131.

Kitchen, D., A. Richardella, J.-M. Tang, M. E. Flatt, and A. Yazdani, 2006, Nature (London) 442, 436.

Kitchen, D.S., 2006, Atom-by-Atom Substitution of Transition metals in GaAs and Visualization of Hole-mediated interactions (University of Illinois at Urbana-Champaign, Champaign).

Klinovaja, J., P. Stano, A. Yazdani, and D. Loss, 2013, Phys. Rev. Lett. 111, 186805.

Komeda, T., 2005, Prog. Surf. Sci. 78, 41.

Kügel, J., P.-J. Hsu, M. Böhme, K. Schneider, J. Senkpiel, D. Serrate, M. Bode, and N. Lorente, 2018, Phys. Rev. Lett. 121, 226402.

Kurz, P., G. Bihlmayer, K. Hirai, and S. Blügel, 2001, Phys. Rev. Lett. 86, 1106.

Lászlóffy, A., L. Rózsa, K. Palotás, L. Udvardi, and L. Szunyogh, 2019, Phys. Rev. B 99, 184430.

Levy, P. M., and A. Fert, 1981, Phys. Rev. B 23, 4667.

Li, J., H. Chen, I. K. Drozdov, A. Yazdani, B. A. Bernevig, and A. H. MacDonald, 2014, Phys. Rev. B 90, 235433.

Lorente, N., and J.-P. Gauyacq, 2009, Phys. Rev. Lett. 103, 176601.

Lorente, N., R. Rurali, and H. Tang, 2005, J. Phys. Condens. Matter 17, S1049.

Loth, S., S. Baumann, C. P. Lutz, D. M. Eigler, and A. J. Heinrich, 2012, Science 335, 196.

Loth, S., M. Etzkorn, C. P. Lutz, E. D. M, and A. J. Heinrich, 2010, Science 329, 1628.

Loth, S., C. P. Lutz, and A. J. Heinrich, 2010, New J. Phys. 12, 125021.

Loth, S., K. von Bergmann, M. Ternes, A. F. Otte, C. P. Lutz, and A. J. Heinrich, 2010, Nat. Phys. 6, 340.

Lutchyn, R. M., E. P. A. M. Bakkers, L. P. Kouwenhoven, P. Krogstrup, C. M. Marcus, and Y. Oreg, 2018, Nat. Rev. Mater. 3, 52 .

Marchukov, O. V., A. G. Volosniev, M. Valiente, D. Petrosyan, and N. T. Zinner, 2016, Nat. Commun. 7, 13070.

Martin, I., and A. F. Morpurgo, 2012, Phys. Rev. B 85, 144505.
Matsui, T., C. Meyer, L. Sacharow, J. Wiebe, and R. Wiesendanger, 2007, Phys. Rev. B 75, 165405.

Mattis, D.C., 2006, Theory of Magnetism Made Simple: An Introduction to Physical Concepts and to Some Useful Mathematical Methods (World Scientific Press, Singapore).

Meier, F., L. Zhou, J. Wiebe, and R. Wiesendanger, 2008, Science 320, 82.

Meir, Y., and N. S. Wingreen, 1992, Phys. Rev. Lett. 68, 2512.

Ménard, G. C., et al., 2015, Nat. Phys. 11, 1013.

Menzel, M., A. Kubetzka, K. von Bergmann, and R. Wiesendanger, 2014, Phys. Rev. Lett. 112, 047204.

Menzel, M., Y. Mokrousov, R. Wieser, J. E. Bickel, E. Vedmedenko, S. Blügel, S. Heinze, K. von Bergmann, A. Kubetzka, and R. Wiesendanger, 2012, Phys. Rev. Lett. 108, 197204.

Mermin, N. D., 2007, Quantum Computer Science: an Introduction (Cambridge University Press, Cambridge, England).

Mermin, N. D., and H. Wagner, 1966, Phys. Rev. Lett. 17, 1133.

Meyer, C., J. Klijn, M. Morgenstern, and R. Wiesendanger, 2003, Phys. Rev. Lett. 91, 076803.

Morgenstern, K., N. Lorente, and K.-H. Rieder, 2013, Phys. Status Solidi (b) 250, 1671.

Morgenstern, M., 2003, Surf. Rev. Lett. 10, 933.

Moriya, T., 1960, Phys. Rev. 120, 91.

Mourigal, M., M. Enderle, A. Klöpperpieper, J.-S. Caux, A. Stunault, and H. M. Rønnow, 2013, Nat. Phys. 9, 435.

Mourik, V., K. Zuo, S. M. Frolov, S. R. Plissard, E. P. A. M. Bakkers, and L. P. Kouwenhoven, 2012, Science 336, 1003.

Müller, G., H. Thomas, H. Beck, and J. C. Bonner, 1981, Phys. Rev. B 24, 1429.

Murmann, S., F. Deuretzbacher, G. Zürn, J. Bjerlin, S. M. Reimann, L. Santos, T. Lompe, and S. Jochim, 2015, Phys. Rev. Lett. 115, 215301.

Nadj-Perge, S., I. K. Drozdov, B. A. Bernevig, and A. Yazdani, 2013, Phys. Rev. B 88, 020407.

Nadj-Perge, S., I. K. Drozdov, J. Li, H. Chen, S. Jeon, J. Seo, A. H. MacDonald, B. A. Bernevig, and A. Yazdani, 2014, Science 346, 602.

Nagaoka, K., T. Jamneala, M. Grobis, and M. F. Crommie, 2002, Phys. Rev. Lett. 88, 077205.

Natterer, F. D., K. Yang, W. Paul, P. Willke, T. Choi, T. Greber, A. J. Heinrich, and C. P. Lutz, 2017, Nature (London) 543, 226.

Nayak, C., S. H. Simon, A. Stern, M. Freedman, and S. Das Sarma, 2008, Rev. Mod. Phys. 80, 1083.

Otte, A. F., M. Ternes, K. von Bergmann, S. Loth, H. Brune, C. P. Lutz, C. F. Hirjibehedin, and A. J. Heinrich, 2008, Nat. Phys. 4, 847.

Pan, Y., J. Yang, S. C. Erwin, K. Kanisawa, and S. Fölsch, 2015, Phys. Rev. Lett. 115, 076803.

Pawlak, R., M. Kisiel, J. Klinovaja, T. Meier, S. Kawai, T. Glatzel, D. Loss, and E. Meyer, 2016, npj Quantum Inf. 2, 16035.

Pientka, F., L. I. Glazman, and F. von Oppen, 2013, Phys. Rev. B 88, 155420.

Pientka, F., Y. Peng, L. Glazman, and F. von Oppen, 2015, Phys. Scr. T164, 014008.

Pollmann, F., 2018, Symmetry-protected topological phases in onedimensional systems: Topological Aspects of Condensed Matter Physics-oi (Oxford University Press, New York).

Pollmann, F., E. Berg, A. M. Turner, and M. Oshikawa, 2012, Phys. Rev. B 85, 075125.

Pollmann, F., A. M. Turner, E. Berg, and M. Oshikawa, 2010, Phys. Rev. B 81, 064439.

Rainis, D., and D. Loss, 2012, Phys. Rev. B 85, 174533.

Rau, I. G., et al., 2014, Science 344, 988. 
Renard, J. P., M. Verdaguer, L. P. Regnault, W. A. C. Erkelens, J. Rossat-Mignod, and W. G. Stirling, 1987, Europhys. Lett. 3, 945.

Renard, J.-P., L.-P. Regnault, and M. Verdaguer, 2002, Magnetism: Molecules to Materials I: Models and Experiments (Wiley-VCH, Weinheim, Germany).

Richardella, A., D. Kitchen, and A. Yazdani, 2009, Phys. Rev. B 80, 045318.

Rolf-Pissarczyk, S., S. Yan, L. Malavolti, J. A. J. Burgess, G. McMurtrie, and S. Loth, 2017, Phys. Rev. Lett. 119, 217201.

Ruby, M., B. W. Heinrich, Y. Peng, F. von Oppen, and K. J. Franke, 2017, Nano Lett. 17, 4473.

Ruby, M., B. W. Heinrich, Y. Peng, F. von Oppen, and K. J. Franke, 2018, Phys. Rev. Lett. 120, 156803.

Ruby, M., et al., 2015, Phys. Rev. Lett. 115, 197204.

Ruby, M., et al., 2016, Phys. Rev. Lett. 117, 186801.

Rudenko, A. N., V. V. Mazurenko, V. I. Anisimov, and A. I. Lichtenstein, 2009, Phys. Rev. B 79, 144418.

Ruderman, M. A., and C. Kittel, 1954, Phys. Rev. 96, 99.

Rudowicz, C., and C. Y. Chung, 2004, J. Phys. Condens. Matter 16, 5825.

Rusinov, A. I., 1969, Sov. J. Exp. Theor. Phys. 29, 1101 [http://www .jetp.ac.ru/cgi-bin/e/index/e/29/6/p1101?a=list].

Sahling, S., G. Remenyi, C. Paulsen, P. Monceau, V. Saligrama, C. Marin, A. Revcolevschi, L. P. Regnault, S. Raymond, and J.E. Lorenzo, 2015, Nat. Phys. 11, 255.

Sakano, M., K. Okawa, M. Kanou, H. Sanjo, T. Okuda, T. Sasagawa, and K. Ishizaka, 2015, Nat. Commun. 6, 8595.

Schecter, M., K. Flensberg, M. H. Christensen, B. M. Andersen, and J. Paaske, 2016, Phys. Rev. B 93, 140503.

Schuh, T., T. Balashov, T. Miyamachi, A. F. Takacs, S. Suga, and W. Wulfhekel, 2010, J. Appl. Phys. 107, 09E156.

Schweflinghaus, B., B. Zimmermann, M. Heide, G. Bihlmayer, and S. Blügel, 2016, Phys. Rev. B 94, 024403.

Serrate, D., P. Ferriani, Y. Yoshida, S. W. Hla, M. Menzel, K. von Bergmann, S. Heinze, A. Kubetzka, and R. Wiesendanger, 2010, Nat. Nanotechnol. 5, 350.

Shiba, H., 1968, Prog. Theor. Phys. 40, 435.

Simon, J., W. S. Bakr, R. Ma, M. E. Tai, P. M. Preiss, and M. Greiner, 2011, Nature (London) 472, 307.

Sklyanin, E. K., and L. D. Faddeev, 1978, Dokl. Akad. Nauk Ser. Fiz. 243, 1430 [Sov. Phys. Dokl. 23, 902 (1978)] [http://inspirehep.net/ record/138384? ln=en].

Smith, D., 1976, J. Magn. Magn. Mater. 1, 214.

Snijders, P. C., and H. H. Weitering, 2010, Rev. Mod. Phys. 82, 307.

Spinelli, A., B. Bryant, F. Delgado, J. Fernández-Rossier, and A. F. Otte, 2014, Nat. Mater. 13, 782.

Spinelli, A., M. P. Rebergen, and A. F. Otte, 2015, J. Phys. Condens. Matter 27, 243203.

Steinbrecher, M., R. Rausch, K. T. That, J. Hermenau, A. A. Khajetoorians, M. Potthoff, R. Wiesendanger, and J. Wiebe, 2018, Nat. Commun. 9, 2853.

Stern, A., 2010, Nature (London) 464, 187.

Stern, A., and N. H. Lindner, 2013, Science 339, 1179.

Stevens, K. W. H., 1952, Proc. Phys. Soc. London Sect. A 65, 209.

Stipe, B. C., M. A. Rezaei, and W. Ho, 1998, Science 280, 1732.

Takhtajan, L. A., 1981, Physica D (Amsterdam) 3, 231.
Tasaki, H., 1991, Phys. Rev. Lett. 66, 798.

Ternes, M., 2015, New J. Phys. 17, 063016.

Ternes, M., A. J. Heinrich, and W.-D. Schneider, 2009, J. Phys. Condens. Matter 21, 053001.

Tersoff, J., and D. R. Hamann, 1985, Phys. Rev. B 31, 805.

Tinkham, M., 2004, Introduction to Superconductivity (Dover, New York).

Tokura, Y., M. Kawasaki, and N. Nagaosa, 2017, Nat. Phys. 13, 1056.

Toskovic, R., R. van den Berg, A. Spinelli, I. S. Eliens, B. van den Toorn, B. Bryant, J.-S. Caux, and A. F. Otte, 2016, Nat. Phys. 12, 656.

Turner, A. M., F. Pollmann, and E. Berg, 2011, Phys. Rev. B 83, 075102.

Udvardi, L., L. Szunyogh, K. Palotás, and P. Weinberger, 2003, Phys. Rev. B 68, 104436.

Ugeda, M. M., et al., 2016, Nat. Phys. 12, 92.

Urdaniz, M. C., M. A. Barral, and A. M. Llois, 2012, Phys. Rev. B 86, 245416.

van Houselt, A., J. Schäfer, H. J. W. Zandvliet, and R. Claessen, 2013, J. Phys. Condens. Matter 25, 010301.

Vazifeh, M. M., and M. Franz, 2013, Phys. Rev. Lett. 111, 206802.

Volosniev, A. G., D. Petrosyan, M. Valiente, D. V. Fedorov, A. S. Jensen, and N. T. Zinner, 2015, Phys. Rev. A 91, 023620.

von Bergmann, K., A. Kubetzka, O. Pietzsch, and R. Wiesendanger, 2014, J. Phys. Condens. Matter 26, 394002.

Wagner, G., and S. Friedberg, 1964, Phys. Lett. 9, 11.

Watanabe, T., and T. Haseda, 1958, J. Chem. Phys. 29, 1429.

Wiebe, J., C. Meyer, J. Klijn, M. Morgenstern, and R. Wiesendanger, 2003, Phys. Rev. B 68, 041402.

Wiebe, J., L. Zhou, and R. Wiesendanger, 2011, J. Phys. D 44, 464009 .

Wiesendanger, R., 2009, Rev. Mod. Phys. 81, 1495.

Xi, X., L. Zhao, Z. Wang, H. Berger, L. Forr, J. Shan, and K. F. Mak, 2015, Nat. Nanotechnol. 10, 765.

Yamada, T., 1969, Prog. Theor. Phys. 41, 880.

Yan, S., D.-J. Choi, J. A. J. Burgess, S. Rolf-Pissarczyk, and S. Loth, 2015, Nat. Nanotechnol. 10, 40.

Yang, J., C. Nacci, J. Martínez-Blanco, K. Kanisawa, and S. Fölsch, 2012, J. Phys. Condens. Matter 24, 354008.

Yang, L., and X. Cui, 2016, Phys. Rev. A 93, 013617.

Yang, L., X. Guan, and X. Cui, 2016, Phys. Rev. A 93, 051605.

Yazdani, A., B. A. Jones, C. P. Lutz, M. F. Crommie, and D. M. Eigler, 1997, Science 275, 1767.

Yosida, K., 1957, Phys. Rev. 106, 893.

Yosida, K., 2001, Theory of Magnetism (Springer Series in Solid-State Sciences) (Springer, New York).

Yu, L., 1965, Acta Phys. Sin. 21, 75.

Zhang, H., et al., 2018, Nature (London) 556, 74.

Zhang, W.-X., R. Ishikawa, B. Breedlove, and M. Yamashita, 2013, RSC Adv. 3, 3772.

Zhang, Y.-h., S. Kahle, T. Herden, C. Stroh, M. Mayor, U. Schlickum, M. Ternes, P. Wahl, and K. Kern, 2013, Nat. Commun. 4, 2110.

Zhou, L., J. Wiebe, S. Lounis, E. Vedmedenko, F. Meier, S. Blügel, P. H. Dederichs, and R. Wiesendanger, 2010, Nat. Phys. 6, 187. 